\title{
The Transmission Dynamics of Hepatitis B Virus via the Fractional-Order Epidemiological Model
}

\author{
Tahir Khan, ${ }^{1,2,3}$ Zi-Shan Qian, ${ }^{4}$ Roman Ullah, ${ }^{5}$ Basem Al Alwan, ${ }^{6}$ Gul Zaman, \\ Qasem M. Al-Mdallal $₫$, ${ }^{7}$ Youssef El Khatib $\oplus^{7},{ }^{7}$ and Khaled Kheder ${ }^{8}$ \\ ${ }^{1}$ Department of Computing, Muscat College, Muscat, Oman \\ ${ }^{2}$ Department of Mathematics, University of Malakand, Dir (L), Chakdara, Pakhtunkhwa, Pakistan \\ ${ }^{3}$ Department of Mathematics and Statistics, Woman University Swabi, Khyber Pakhtunkhwa, Pakistan \\ ${ }^{4}$ School of Physical Sciences, University of California, Irvine, California 92617, USA \\ ${ }^{5}$ General Requirements Department, CAS Sohar, , University of Technology and Applied Sciences, Muscat, Oman \\ ${ }^{6}$ Chemical Engineering Department, College of Engineering, King Khalid University, Abha 61411, Saudi Arabia \\ ${ }^{7}$ Department of Mathematical Sciences, UAE University, P.O. Box 15551, Al-Ain, UAE \\ ${ }^{8}$ Civil Engineering Department, College of Engineering, King Khalid University, Abha 61411, Saudi Arabia
}

Correspondence should be addressed to Qasem M. Al-Mdallal; q.almdallal@uaeu.ac.ae

Received 6 September 2021; Accepted 2 November 2021; Published 27 December 2021

Academic Editor: Mustafa Cagri Kutlu

Copyright (c) 2021 Tahir Khan et al. This is an open access article distributed under the Creative Commons Attribution License, which permits unrestricted use, distribution, and reproduction in any medium, provided the original work is properly cited.

We investigate and analyze the dynamics of hepatitis B with various infection phases and multiple routes of transmission. We formulate the model and then fractionalize it using the concept of fractional calculus. For the purpose of fractionalizing, we use the Caputo-Fabrizio operator. Once we develop the model under consideration, existence and uniqueness analysis will be discussed. We use fixed point theory for the existence and uniqueness analysis. We also prove that the model under consideration possesses a bounded and positive solution. We then find the basic reproductive number to perform the steady-state analysis and to show that the fractional-order epidemiological model is locally and globally asymptotically stable under certain conditions. For the local and global analysis, we use linearization, mean value theorem, and fractional Barbalat's lemma, respectively. Finally, we perform some numerical findings to support the analytical work with the help of graphical representations.

\section{Introduction}

Hepatitis B virus causes inflammation of the liver. It results from a noncytopathic virus which is called the hepatitis B virus $(\mathrm{HBV})$. Characteristic of $\mathrm{HBV}$ is its high tissue and species specificity, as well as a unique genomic organization and replication mechanism. The infection of $\mathrm{HBV}$ has multiple phases: acute and chronic. The acute one refers to the first six months whenever there is an exposure of some one to the virus. Usually, in this period, the immune system has the capability to vanish the infection, while for some severe cases, it may also lead to the serious stage and so results in the lifelong illness. This is also known as the chronic stage. It could be noted that whenever HBsAg is positive for a person for a period of more than 6 months, it shows that it has a chronic illness. In case of the chronic stage, often, the individual has no history of the acute stage. This infection may also lead to the scarring of the liver, become liver failure, and produce liver cancer [1]. Hepatitis B virus is transferred by many ways: blood (razors, sharing of blades, tooth brushes, etc.) and semen and vaginal [2-5]. One of the other key sources of transmission is from the infected mother to her child called vertical transmission [6]. Worldwide, there are millions of infected population according to the $\mathrm{WHO}$, in which only 93 millions are infected in China [7, 8]. Vaccines are available to immunize from the $\mathrm{HBV}$ which are very effective and almost provide permanent immunity $[9,10]$.

Mathematical modeling of infectious diseases has a vast field and has a rich literature, which plays a significant role to explore the dynamics and suggest the control mechanism. Since hepatitis B is one of the life-threatening and leading causes of death, it obtained the attention of various researches, 
and consequently, many epidemiological models were developed (see [11-15]). Anderson and May presented a study in the form of a simple model to investigate the influence of carriers on the transmission of hepatitis B [16]. Williams et al. presented and analyzed the hepatitis $\mathrm{B}$ dynamics in the United Kingdom [17]. Moreover, a model was presented by Medley et al. to forecast a mechanism for eliminating hepatitis B from New Zealand [18]. In a similar way, a model that evaluates the effectiveness of the vaccination programme with the effect of age in China was presented by Zhao et al. [19]. Bakare et al. proposed the analysis of control by using an SIR epidemic model [20]. More epidemic models were investigated with control strategies by Kamyad et al. [21]. Onyango developed a model to study the multiple endemic solutions [22]. Similarly, Zhang et al. studied the dynamics of hepatitis B in Xinjiang [23]. Very recently, Khan et al. $[24,25]$ and Nana-Kyere et al. [26] formulated some epidemiological models to study different parameters' influences on the disease transmission and to suggest some control measures for the elimination of the infection. The study of fractional calculus obtained the attention of researchers and is growing rapidly. This analysis has been used to capture the axioms of inherited and the memory of various natural and physical phenomena occurring in different fields of science and technology. Numerous classical models have been proved with less accuracy in case of predicting the future dynamics of a system. However, models having fractional order are more useful to allocate and detain the missing information $[27,28]$. It could also be stated that the classical derivative does not provide the dynamics between two different points $[29,30]$.

It is noted that hepatitis B virus transmission is influenced by different factors, i.e., various phases, routes of transmission, etc. Especially, the carriers are significant. The chronic carriers have no symptoms while transmitting the infection. Moreover, it could also be noted that the increased development of fractional calculus and fractional-order epidemiological models are more suitable than the classical order epidemic models and complex dynamics of hepatitis $B$; we therefore investigate a hepatitis $B$ virus transmission epidemic model with various infection phases and multiple routes of transmission. Moreover, we also use the fractional calculus to fractionalize the model under consideration which has not yet been studied to the best of our knowledge. Once we formulate the model, we then study the existence analysis as well as uniqueness to prove the well-posedness and biological feasibility of the problem under consideration. For this analysis, the fixed point theory will be used. We also prove that the solutions of the proposed system are bounded and positive. We then discuss the steady state of the proposed model and investigate that the model under consideration is locally and globally asymptotically stable. For local stability analysis, we use the method of linearization, mean value theorem, and fractional Barbalat's lemma. Finally, some numerical simulations will be performed to support the analytical work and show the difference between the classical and fractional order.

\section{Preliminaries}

Here, we describe the fundamental concepts related to the fractional calculus which are helpful to obtain our results.

Definition 1 (see [30]). Let us assume a function $\varphi(t)$ such that $\phi \in H^{1}(0, T), T>0$; if $\alpha>0$ and $n-1<\alpha<n, n \in \mathbb{N}$, then the Caputo and Caputo-Fabrizio derivative of the fractional order $(\alpha)$ are defined, respectively, as

$$
{ }^{C} D_{0, t}^{\alpha}\{\varphi(t)\}=\frac{1}{\Gamma(n-\alpha)} \int_{0}^{t}(t-x)^{n-\alpha-1} \varphi^{n}(x) \mathrm{d} x
$$

and

$$
{ }^{C F} D_{0, t}^{\alpha}\{\varphi(t)\}=\frac{K(\alpha)}{(1-\alpha)} \int_{0}^{t} \varphi^{\prime}(y) \exp \left(\frac{\alpha(y-t)}{1-\alpha}\right) \mathrm{d} y .
$$

In equations (1) and (2), $C$ and $C F$ represent, respectively, Caputo and Caputo-Fabrizio, while $t>0$ and $K(\alpha)$ represent the normalization function such that $K(1)=0=K(0)$.

Definition 2 (see [30]). If $0<\alpha<1$ and $\varphi(t)$ varies with time $t$, then the Riemann-Liouville integral of order $(\alpha)$ is defined as

$$
{ }^{R L} J_{0, t}^{\alpha}\{\varphi(t)\}=\frac{1}{\Gamma(\alpha)} \int_{0}^{t}(t-y)^{\alpha-1} \varphi(y) \mathrm{d} y,
$$

while the integral of order $(\alpha)$ in the Caputo-FabrizioCaputo $(C F)$ sense is defined by

$$
{ }^{C F} J_{0, t}^{\alpha}\{\varphi(t)\}=\frac{2(1-\alpha) \varphi(t)}{(2-\alpha) K(\alpha)}+\frac{2 \alpha}{(2-\alpha) K(\alpha)} \int_{0}^{t} \varphi(y) \mathrm{d} y .
$$

\section{Model Formulation}

We formulate the model keeping in view the characteristics of hepatitis B virus and so distribute the total population symbolized by $T(t)$ into different compartmental population sizes, i.e., susceptible $S(t)$, acute $A(t)$, chronic $C(t)$, recovered/immune $R(t)$, and vaccinated $V(t)$. We also define some constraints for the proposed problem:

$a_{1}$ : all the variables $(S, A, C, R$, and $V)$ and the parameters $(\Pi, \zeta, \beta, \rho, \vartheta, \eta, \sigma, p, \gamma, \varepsilon$, and $\tau)$ are nonnegative in the epidemic problem that is under consideration.

$a_{2}$ : the successfully vaccinated portion $\eta$ of the susceptible individuals goes to the recovered class.

$a_{3}$ : the contact of susceptible with acute infected as well as with chronically infected is, respectively, denoted by $\beta$ and $\rho \beta$, which lead to the acute portion with probability $p$ and go to chronic with probability $(1-p)$, where this assumption is based on the hypothesis that some of the individuals have no history of acute illness. 
$a_{4}$ : since some of the individuals got recovery in the acute stage and it leads to the chronic stage for some severe cases, therefore, a natural recovery with probability $q$ has been proposed, while $(1-q)$ leads to the chronic stage.

$a_{5}$ : the recovery under treatment $(\tau)$ is taken of the chronic population. $a_{6}$ : the disease-induced death rate $(\varepsilon)$ occurs in the chronic stage only.

$a_{7}$ : the newborn rate is $\Pi$ and assumed to be susceptible, while getting successful vaccination $(\zeta)$ leads to the vaccinated class.

In light of these assumptions, we develop a model as presented in the following:

$$
\left\{\begin{array}{l}
\frac{\mathrm{d} S(t)}{\mathrm{d} t}=(1-\zeta) \Pi-\beta S(t) A(t)-\rho \beta S(t) C(t)-(\vartheta+\eta) S(t)+\sigma V(t), \\
\frac{\mathrm{d} A(t)}{\mathrm{d} t}=p\{\beta S(t) A(t)+\rho \beta S(t) C(t)\}-(\vartheta+\gamma) A(t), \\
\frac{\mathrm{d} C(t)}{\mathrm{d} t}=(1-p)\{\beta S(t) A(t)+\rho \beta S(t) C(t)\}+q \gamma A(t)-(\vartheta+\varepsilon+\tau) C(t), \\
\frac{\mathrm{d} R(t)}{\mathrm{d} t}=(1-q) \gamma A(t)+\eta S(t)+\tau C(t)-\vartheta R(t), \\
\frac{\mathrm{d} V(t)}{\mathrm{d} t}=\zeta \Pi-(\vartheta+\sigma) V(t)
\end{array}\right.
$$

with initial population sizes

$$
S(0)>0, A(0) \geq 0, C(0) \geq 0, R(0)>0, V(0)>0,
$$

where $\zeta$ is the proportion of successful vaccination individuals and $\Pi$ is the newborn rate. Similarly, the transmission rate of hepatitis $\mathrm{B}$ is denoted by $\beta$, while the reduced transmission rate is $\rho$. Moreover, $\vartheta$ and $\eta$ are, respectively, the natural death rate and permanent recovered individuals' rate. We also symbolize the recovery rate of acute and chronic hepatitis B individuals by $\gamma$ and $\tau$, respectively. The disease-induced death rate is represented by $\varepsilon$, while those individuals who lose their immunity are represented by $\sigma$.

We extend the reported model by equation (5) to the associated fractional-order $(\alpha<0<\alpha<1)$ version by taking into account the Caputo-Fabrizio-Caputo $(C F)$ operator. We therefore replace the derivatives in the problem under consideration with a fractional derivative to maintain the dimension of both sides of the equations of the proposed model taking the $\alpha$ power of each parameter which becomes

$$
\left\{\begin{array}{l}
{ }^{C F} D_{0, t}^{\alpha}(S(t))=\left(1-\zeta^{\alpha}\right) \Pi^{\alpha}-\beta^{\alpha} S(t) A(t)-\rho^{\alpha} \beta^{\alpha} S(t) C(t)-\left(\vartheta^{\alpha}+\eta^{\alpha}\right) S(t)+\sigma^{\alpha} V(t), \\
{ }^{C F} D_{0, t}^{\alpha}(A(t))=p\left\{\beta^{\alpha} S(t) A(t)+\rho^{\alpha} \beta^{\alpha} S(t) C(t)\right\}-\left(\vartheta^{\alpha}+\gamma^{\alpha}\right) A(t), \\
{ }^{C F} D_{0, t}^{\alpha}(C(t))=(1-p)\left\{\beta^{\alpha} S(t) A(t)+\rho^{\alpha} \beta^{\alpha} S(t) C(t)\right\}+q \gamma^{\alpha} A(t)-\left(\vartheta^{\alpha}+\epsilon^{\alpha}+\tau^{\alpha}\right) C(t), \\
{ }^{C F} D_{0, t}^{\alpha}(R(t))=(1-q) \gamma^{\alpha} A(t)+\eta^{\alpha} S(t)+\tau^{\alpha} C(t)-\vartheta^{\alpha} R(t) \\
{ }^{C F} D_{0, t}^{\alpha}(V(t))=\zeta^{\alpha} \Pi^{\alpha}-\left(\vartheta^{\alpha}+\sigma^{\alpha}\right) V(t) .
\end{array}\right.
$$

We now discuss the existence and uniqueness of the above fractional-order epidemiological model (7) in the following section. 


\section{Existence and Uniqueness}

This section is devoted to the existence and uniqueness analysis of the solution of fractional-order epidemiological model (7). We use the concept of fixed point theory and prove the solution existence and uniqueness. For this analysis, transforming the proposed system into an integral equation, we obtain

$$
\begin{aligned}
& S(t)=S(0)+{ }^{C F} J_{0, t}^{\alpha}\left\{\left(1-\zeta^{\alpha}\right) \Pi^{\alpha}-\beta^{\alpha} S(t) A(t)-\rho^{\alpha} \beta^{\alpha} S(t) C(t)-\left(\vartheta^{\alpha}+\eta^{\alpha}\right) S(t)+\sigma^{\alpha} V(t)\right\}, \\
& A(t)=A(0)+{ }^{C F} J_{0, t}^{\alpha}\left\{p\left\{\beta^{\alpha} S(t) A(t)+\rho^{\alpha} \beta^{\alpha} S(t) C(t)\right\}-\left(\vartheta^{\alpha}+\gamma^{\alpha}\right) A(t)\right\}, \\
& C(t)=C(0)+{ }^{C F} J_{0, t}^{\alpha}\left\{(1-p)\left\{\beta^{\alpha} S(t) A(t)+\rho^{\alpha} \beta^{\alpha} S(t) C(t)\right\}+q \gamma^{\alpha} A(t)-\left(\vartheta^{\alpha}+\varepsilon^{\alpha}+\tau^{\alpha}\right) C(t)\right\}, \\
& R(t)=R(0)+{ }^{C F} J_{0, t}^{\alpha}\left\{(1-q) \gamma^{\alpha} A(t)+\eta^{\alpha} S(t)+\tau^{\alpha} C(t)-\vartheta^{\alpha} R(t)\right\}, \\
& V(t)=V(0)+{ }^{C F} J_{0, t}^{\alpha}\left\{\zeta^{\alpha} \Pi^{\alpha}-\left(\vartheta^{\alpha}+\sigma^{\alpha}\right) V(t)\right\} .
\end{aligned}
$$

Taking the CF fractional integral of both sides of the above system leads to the assertions as given in the following:

$$
\begin{aligned}
S(t)= & S(0)+\frac{2(1-\alpha)}{K(\alpha)(2-\alpha)}\left\{\left(1-\varsigma^{\alpha}\right) \Pi^{\alpha}-\beta^{\alpha} S(y) A(y)-\rho^{\alpha} \beta^{\alpha} S(t) C(t)-\left(\vartheta^{\alpha}+\eta^{\alpha}\right) S(t)+\sigma^{\alpha} V(t)\right\} \\
& +\frac{2 \alpha}{K(\alpha)(2-\alpha)} \int_{0}^{t}\left\{\left(1-\varsigma^{\alpha}\right)\right\}-\beta^{\alpha} S(y) A(y)-\rho^{\alpha} \beta^{\alpha} S(y) C(y)-\left(\vartheta^{\alpha}+\eta^{\alpha}\right) S(y)+\sigma^{\alpha} V(y) d y, \\
A(t)= & A(0)+\frac{2(1-\alpha)}{K(\alpha)(2-\alpha)}\left\{p\left\{\beta^{\alpha} S(t) A(t)+p^{\alpha} \beta^{\alpha} S(t) C(t)\right\}-\left(\vartheta^{\alpha}+\gamma^{\alpha}\right) S(t)\right\} \\
& +\frac{2 \alpha}{K(\alpha)(2-\alpha)} \int_{0}^{t}\left\{p\left\{\beta^{\alpha} S(y) A(y)+p^{\alpha} \beta^{\alpha} S(y) C(y)\right\}-\left(\vartheta^{\alpha}+\gamma^{\alpha}\right) A(y)\right\} d y, \\
B(t)= & B(0)+\frac{2(1-\alpha)}{K(\alpha)(2-\alpha)}\left\{(1-p)\left\{\beta^{\alpha} S(t) A(t)+p^{\alpha} \beta^{\alpha} S(t) C(t)\right\}+q \gamma^{\alpha} A(t)-\left(\vartheta^{\alpha}+\varepsilon^{\alpha}+\tau^{\alpha}\right) C(t)\right\} \\
& +\frac{2 \alpha}{K(\alpha)(2-\alpha)} \int_{0}^{t}\left\{(1-p)\left\{\beta^{\alpha} S(y) A(y)+p^{\alpha} \beta^{\alpha} S(y) C(y)\right\}-q \gamma^{\alpha} A(t)-\left(\vartheta^{\alpha}+\varepsilon^{\alpha}+\tau^{\alpha}\right) C(y)\right\} d y, \\
R(t)= & R(0)+\frac{2(1-\alpha)}{K(\alpha)(2-\alpha)}\left\{(1-q) \gamma^{\alpha} A(t)+\eta^{\alpha} S(t)+\tau^{\alpha} C(t)-\vartheta^{\alpha} R(t)\right\} \\
& +\frac{2 \alpha}{K(\alpha)(2-\alpha)} \int_{0}^{t}\left\{(1-q) \gamma^{\alpha} A(y)+\eta^{\alpha} S(y)+\tau^{\alpha} C(y)-\vartheta^{\alpha} R(y)\right\} d y, \\
V(t)= & V(0)+\frac{2(1-\alpha)}{K(\alpha)(2-\alpha)}\left\{\varsigma^{\alpha} \Pi^{\alpha}-\left(\vartheta^{\alpha}+\sigma^{\alpha}\right) V(t)\right\} \\
& +\frac{2 \alpha}{K(\alpha)(2-\alpha)} \int_{0}^{t}\left\{\varsigma^{\alpha} \Pi^{\alpha}-\left(\vartheta^{\alpha}+\sigma^{\alpha}\right) V(t)\right\} d y .
\end{aligned}
$$


Let $\ell_{1}, \ell_{2}, \ell_{3}, \ell_{4}$, and $\ell_{5}$ be the kernels, and they are defined by

$$
\begin{aligned}
& \ell_{1}(S(t), t)=\left\{\left(1-\zeta^{\alpha}\right) \Pi^{\alpha}-\beta^{\alpha} S(t) A(t)-\rho^{\alpha} \beta^{\alpha} S(t) C(t)-\left(\vartheta^{\alpha}+\eta^{\alpha}\right) S(t)+\sigma^{\alpha} V(t)\right\}, \\
& \ell_{2}(A(t), t)=\left\{p\left\{\beta^{\alpha} S(t) A(t)+\rho^{\alpha} \beta^{\alpha} S(t) C(t)\right\}-\left(\vartheta^{\alpha}+\gamma^{\alpha}\right) A(t)\right\}, \\
& \ell_{3}(C(t), t)=\left\{(1-p)\left\{\beta^{\alpha} S(t) A(t)+\rho^{\alpha} \beta^{\alpha} S(t) C(t)\right\}+q \gamma^{\alpha} A(t)-\left(\vartheta^{\alpha}+\varepsilon^{\alpha}+\tau^{\alpha}\right) C(t)\right\}, \\
& \ell_{4}(R(t), t)=\left\{(1-q) \gamma^{\alpha} A(t)+\eta^{\alpha} S(t)+\tau^{\alpha} C(t)-\vartheta^{\alpha} R(t)\right\}, \\
& \ell_{5}(V(t), t)=\left\{\zeta^{\alpha} \Pi^{\alpha}-\left(\vartheta^{\alpha}+\sigma^{\alpha}\right) V(t)\right\} .
\end{aligned}
$$

Theorem 1. The above kernels $\ell_{1}, \ell_{2}, \ell_{3}, \ell_{4}$, and $\ell_{5}$ satisfy axioms of Lipschitz conditions.
Proof. Let us assume that $S$ and $S_{1}, A$ and $A_{1}, C$ and $C_{1}, R$ and $R_{1}$, and $V$ and $V_{1}$ are, respectively, the two functions for the kernels $\ell_{1}, \ell_{2}, \ell_{3}, \ell_{4}$, and $\ell_{5}$, so we establish the following system:

$$
\begin{aligned}
\ell_{1}(S(t), t)-\ell_{1}\left(S_{1}(t), t\right) & =\left\{\left(1-\zeta^{\alpha}\right) \Pi^{\alpha}-\beta^{\alpha}\left(S-S_{1}\right) A(t)-\rho^{\alpha} \beta^{\alpha}\left(S-S_{1}\right) C(t)-\left(\vartheta^{\alpha}+\eta^{\alpha}\right)\left(S-S_{1}\right)+\sigma^{\alpha} V(t)\right\}, \\
\ell_{2}(A(t), t)-\ell_{2}\left(A_{1}(t), t\right) & =\left\{p\left\{\beta^{\alpha} S(t)\left(A-A_{1}\right)+\rho^{\alpha} \beta^{\alpha} S(t) C(t)\right\}-\left(\vartheta^{\alpha}+\gamma^{\alpha}\right)\left(A-A_{1}\right)\right\}, \\
\ell_{3}(C(t), t)-\ell_{3}\left(C_{1}(t), t\right) & =\left\{(1-p)\left\{\beta^{\alpha} S(t) A(t)+\rho^{\alpha} \beta^{\alpha} S(t)\left(C-C_{1}\right)\right\}+q \gamma^{\alpha} A(t)-\left(\vartheta^{\alpha}+\varepsilon^{\alpha}+\tau^{\alpha}\right) C(t)\right\}, \\
\ell_{4}(R(t), t)-\ell_{4}\left(R_{1}(t), t\right) & =\left\{(1-q) \gamma^{\alpha} A(t)+\eta^{\alpha} S(t)+\tau^{\alpha} C(t)-\vartheta^{\alpha}\left(R-R_{1}\right)\right\}, \\
\ell_{5}(V(t), t)-\ell_{5}\left(V_{1}(t), t\right) & =\left\{\zeta^{\alpha} \Pi^{\alpha}-\left(\vartheta^{\alpha}+\sigma^{\alpha}\right)\left(V-V_{1}\right)\right\} .
\end{aligned}
$$

Cauchy's inequality application leads to the following system:

$$
\begin{aligned}
\left\|\ell_{1}(S(t), t)-\ell_{1}\left(S_{1}(t), t\right)\right\| & =\left\|\left(1-\zeta^{\alpha}\right) \Pi^{\alpha}-\beta^{\alpha}\left(S-S_{1}\right) A(t)-\rho^{\alpha} \beta^{\alpha}\left(S-S_{1}\right) C(t)-\left(\vartheta^{\alpha}+\eta^{\alpha}\right)\left(S-S_{1}\right)+\sigma^{\alpha} V(t)\right\|, \\
\left\|\ell_{2}(A(t), t)-\ell_{2}\left(A_{1}(t), t\right)\right\| & =\left\|p\left\{\beta^{\alpha} S(t)\left(A-A_{1}\right)+\rho^{\alpha} \beta^{\alpha} S(t) C(t)\right\}-\left(\vartheta^{\alpha}+\gamma^{\alpha}\right)\left(A-A_{1}\right)\right\|, \\
\left\|e_{3}(C(t), t)-\ell_{3}\left(C_{1}(t), t\right)\right\| & =\left\|(1-p)\left\{\beta^{\alpha} S(t) A(t)+\rho^{\alpha} \beta^{\alpha} S(t)\left(C-C_{1}\right)\right\}+q \gamma^{\alpha} A(t)-\left(\vartheta^{\alpha}+\varepsilon^{\alpha}+\tau^{\alpha}\right) C(t)\right\|, \\
\left\|e_{4}(R(t), t)-\ell_{4}\left(R_{1}(t), t\right)\right\| & =\left\|(1-q) \gamma^{\alpha} A(t)+\eta^{\alpha} S(t)+\tau^{\alpha} C(t)-\vartheta^{\alpha}\left(R-R_{1}\right)\right\| \\
\left\|\ell_{5}(V(t), t)-\ell_{5}\left(V_{1}(t), t\right)\right\| & =\left\|\zeta^{\alpha} \Pi^{\alpha}-\left(\vartheta^{\alpha}+\sigma^{\alpha}\right)\left(V-V_{1}\right)\right\| .
\end{aligned}
$$

Recursively, we obtain

$$
\begin{aligned}
& S(t)=\frac{2(1-\alpha) \ell_{1}\left(S_{n-1}(t), t\right)}{(2-\alpha) K(\alpha)}+\frac{2 \alpha}{(2-\alpha) K(\alpha)} \int_{0}^{t} \ell_{1}\left(S_{n-1}(y), y\right) \mathrm{d} y, \\
& A(t)=\frac{2(1-\alpha) \ell_{2}\left(A_{n-1}(t), t\right)}{(2-\alpha) K(\alpha)}+\frac{2 \alpha}{(2-\alpha) K(\alpha)} \int_{0}^{t} \ell_{2}\left(A_{n-1}(y), y\right) \mathrm{d} y \\
& C(t)=\frac{2(1-\alpha) \ell_{3}\left(C_{n-1}(t), t\right)}{(2-\alpha) K(\alpha)}+\frac{2 \alpha}{(2-\alpha) K(\alpha)} \int_{0}^{t} \ell_{3}\left(C_{n-1}(y), y\right) \mathrm{d} y \\
& R(t)=\frac{2(1-\alpha) \ell_{4}\left(R_{n-1}(t), t\right)}{(2-\alpha) K(\alpha)}+\frac{2 \alpha}{(2-\alpha) K(\alpha)} \int_{0}^{t} \ell_{4}\left(R_{n-1}(y), y\right) \mathrm{d} y \\
& V(t)=\frac{2(1-\alpha) \ell_{5}\left(V_{n-1}(t), t\right)}{(2-\alpha) K(\alpha)}+\frac{2 \alpha}{(2-\alpha) K(\alpha)} \int_{0}^{t} \ell_{5}\left(V_{n-1}(y), y\right) \mathrm{d} y
\end{aligned}
$$


The norm application with majorizing and the difference between successive terms imply

$$
\begin{aligned}
& \left\|U_{n}(t)\right\|=\left\|S_{n}(t)-S_{1,(n-1)}(t)\right\| \leq \frac{2(1-\alpha)}{K(\alpha)(2-\alpha)}\left\|\ell_{1}\left(S_{n-1}(t), t\right)-\ell_{1}\left(S_{1,(n-2)}(t), t\right)\right\| \\
& +\frac{2(1-\alpha)}{K(\alpha)(2-\alpha)}\left\|\int_{0}^{t}\left[\ell_{1}\left(S_{n-1}(y), y\right)-\ell_{1}\left(S_{1, n-2}(y), y\right)\right] \mathrm{d} y\right\|, \\
& \left\|W_{n}(t)\right\|=\left\|A_{n}(t)-A_{1,(n-1)}(t)\right\| \leq \frac{2(1-\alpha)}{K(\alpha)(2-\alpha)}\left\|\ell_{2}\left(A_{n-1}(t), t\right)-\ell_{2}\left(A_{1,(n-2)}(t), t\right)\right\| \\
& +\frac{2(1-\alpha)}{K(\alpha)(2-\alpha)}\left\|\int_{0}^{t}\left[\ell_{2}\left(A_{n-1}(y), y\right)-\ell_{2}\left(A_{1, n-2}(y), y\right)\right] \mathrm{d} y\right\|, \\
& \left\|X_{n}(t)\right\|=\left\|C_{n}(t)-C_{1,(n-1)}(t)\right\| \leq \frac{2(1-\alpha)}{K(\alpha)(2-\alpha)}\left\|\ell_{3}\left(C_{n-1}(t), t\right)-\ell_{3}\left(C_{1,(n-2)}(t), t\right)\right\| \\
& +\frac{2(1-\alpha)}{K(\alpha)(2-\alpha)}\left\|\int_{0}^{t}\left[\ell_{3}\left(C_{n-1}(y), y\right)-\ell_{3}\left(C_{1, n-2}(y), y\right)\right] \mathrm{d} y\right\|, \\
& \left\|Y_{n}(t)\right\|=\left\|R_{n}(t)-R_{1,(n-1)}(t)\right\| \leq \frac{2(1-\alpha)}{K(\alpha)(2-\alpha)}\left\|\ell_{4}\left(R_{n-1}(t), t\right)-\ell_{4}\left(R_{1,(n-2)}(t), t\right)\right\| \\
& +\frac{2(1-\alpha)}{K(\alpha)(2-\alpha)}\left\|\int_{0}^{t}\left[\ell_{4}\left(R_{n-1}(y), y\right)-\ell_{4}\left(R_{1, n-2}(y), y\right)\right] \mathrm{d} y\right\|, \\
& \left\|Z_{n}(t)\right\|=\left\|V_{n}(t)-S_{1,(n-1)}(t)\right\| \leq \frac{2(1-\alpha)}{K(\alpha)(2-\alpha)}\left\|\ell_{5}\left(V_{n-1}(t), t\right)-\ell_{5}\left(S_{1,(n-2)}(t), t\right)\right\| \\
& +\frac{2(1-\alpha)}{K(\alpha)(2-\alpha)}\left\|\int_{0}^{t}\left[\ell_{4}\left(V_{n-1}(y), y\right)-\ell_{5}\left(V_{1, n-2}(y), y\right)\right] \mathrm{d} y\right\|
\end{aligned}
$$

where

$$
\begin{aligned}
& \sum_{i=0}^{\infty} U_{i}(t)=S_{n}(t), \\
& \sum_{i=0}^{\infty} W_{i}(t)=A_{n}(t), \\
& \sum_{i=0}^{\infty} X_{i}(t)=B_{n}(t), \\
& \sum_{i=0}^{\infty} Y_{i}(t)=R_{n}(t), \\
& \sum_{i=0}^{\infty} Z_{i}(t)=V_{n}(t) .
\end{aligned}
$$

Since the kernels $\ell_{1}, \ldots, \ell_{5}$ satisfy the Lipschitz conditions, 


$$
\begin{aligned}
\left\|U_{n}(t)\right\|= & \left\|S_{n}(t)-S_{1, n-1}(t)\right\| \leq \frac{2(1-\alpha)}{K(\alpha)(2-\alpha)} \tau_{1}\left\|S_{n-1}(t)-S_{1, n-2}(t)\right\| \\
& +\frac{2 \alpha}{(2-\alpha) K(\alpha)} \tau_{2} \int_{0}^{t}\left\|S_{n-1}(y)-S_{1, n-2}(y)\right\| \mathrm{d} y, \\
\left\|W_{n}(t)\right\|= & \left\|A_{n}(t)-A_{1, n-1}(t)\right\| \leq \frac{2(1-\alpha)}{K(\alpha)(2-\alpha)} \tau_{3}\left\|A_{n-1}(t)-A_{1, n-2}(t)\right\| \\
& +\frac{2 \alpha}{(2-\alpha) K(\alpha)} \tau_{4} \int_{0}^{t}\left\|A_{n-1}(y)-A_{1, n-2}(y)\right\| \mathrm{d} y, \\
\left\|X_{n}(t)\right\|= & \left\|C_{n}(t)-C_{1, n-1}(t)\right\| \leq \frac{2(1-\alpha)}{K(\alpha)(2-\alpha)} \tau_{5}\left\|C_{n-1}(t)-C_{1, n-2}(t)\right\| \\
& +\frac{2 \alpha}{(2-\alpha) K(\alpha)} \tau_{6} \int_{0}^{t}\left\|C_{n-1}(y)-C_{1, n-2}(y)\right\| \mathrm{d} y,\left\|Y_{n}(t)\right\| \\
& +\frac{2 \alpha}{(2-\alpha) K(\alpha)} \tau_{10} \int_{0}^{t}\left\|V_{n-1}(y)-V_{1, n-2}(y)\right\| \mathrm{d} y . \\
\left\|Z_{n}(t)\right\|= & \left\|V_{n}(t)-V_{1, n-1}(t)\right\| \leq \frac{2(1-\alpha)}{K(\alpha)(2-\alpha)} \tau_{9}\left\|V_{n-1}(t)-V_{1, n-2}(t)\right\|
\end{aligned}
$$

Theorem 2. The solution of fractional-order epidemiological model (7) exists.
Proof. The use of equation (15) with the recursive scheme implies

$$
\begin{aligned}
& \left\|U_{n}(t)\right\| \leq\|S(0)\|+\left\{\left(\frac{2 \tau_{1}(1-\alpha)}{K(\alpha)(2-\alpha)}\right)^{n}\right\}+\left\{\left(\frac{2 \tau_{2} \alpha t}{K(\alpha)(2-\alpha)}\right)^{n}\right\}, \\
& \left\|W_{n}(t)\right\| \leq\|A(0)\|+\left\{\left(\frac{2 \tau_{3}(1-\alpha)}{K(\alpha)(2-\alpha)}\right)^{n}\right\}+\left\{\left(\frac{2 \tau_{4} \alpha t}{K(\alpha)(2-\alpha)}\right)^{n}\right\}, \\
& \left\|X_{n}(t)\right\| \leq\|(0)\|+\left\{\left(\frac{2 \tau_{5}(1-\alpha)}{K(\alpha)(2-\alpha)}\right)^{n}\right\}+\left\{\left(\frac{2 \tau_{6} \alpha t}{K(\alpha)(2-\alpha)}\right)^{n}\right\}, \\
& \left\|Y_{n}(t)\right\| \leq\|R(0)\|+\left\{\left(\frac{2 \tau_{7}(1-\alpha)}{K(\alpha)(2-\alpha)}\right)^{n}\right\}+\left\{\left(\frac{2 \tau_{8} \alpha t}{K(\alpha)(2-\alpha)}\right)^{n}\right\}, \\
& \left\|Z_{n}(t)\right\| \leq\|V(0)\|+\left\{\left(\frac{2 \tau_{9}(1-\alpha)}{K(\alpha)(2-\alpha)}\right)^{n}\right\}+\left\{\left(\frac{2 \tau_{10} \alpha t}{K(\alpha)(2-\alpha)}\right)^{n}\right\} .
\end{aligned}
$$

We investigate that equation (17) is the solution of model (7); therefore, we make the following substitutions:

$$
\begin{gathered}
S(t)=S_{n}(t)-Y_{1, n}(t), \\
A(t)=A_{n}(t)-Y_{2, n}(t), \\
B(t)=B_{n}(t)-Y_{3, n}(t), \\
R(t)=R_{n}(t)-Y_{4, n}(t), \\
V(t)=V_{n}(t)-Y_{5, n}(t),
\end{gathered}
$$


where $Y_{1, n}(t), Y_{2, n}(t), Y_{3, n}(t), Y_{4, n}(t)$, and $Y_{5, n}(t)$ denote the remainder terms of the series. So,

$$
\begin{aligned}
& S(t)-S_{n-1}(t)=\frac{2(1-\alpha) \ell_{1}\left(S(t)-\Pi_{1, n}(t)\right)}{K(\alpha)(2-\alpha)}+\frac{2 \alpha}{K(\alpha)(2-\alpha)} \int_{0}^{t} \ell_{1}\left(S(y)-Y_{1, n}(y)\right) \mathrm{d} y, \\
& A(t)-A_{n-1}(t)=\frac{2 \ell_{2}\left(A(t)-Y_{2, n}(t)\right)(1-\alpha)}{K(\alpha)(2-\alpha)}+\frac{2 \alpha}{K(\alpha)(2-\alpha)} \int_{0}^{t} \ell_{2}\left(A(y)-Y_{1, n}(y)\right) \mathrm{d} y, \\
& C(t)-C_{n-1}(t)=\frac{2 \ell_{3}\left(C(t)-Y_{2, n}(t)\right)(1-\alpha)}{K(\alpha)(2-\alpha)}+\frac{2 \alpha}{K(\alpha)(2-\alpha)} \int_{0}^{t} \ell_{2}\left(C(y)-Y_{1, n}(y)\right) \mathrm{d} y, \\
& R(t)-R_{n-1}(t)=\frac{2 \ell_{4}\left(R(t)-Y_{2, n}(t)\right)(1-\alpha)}{K(\alpha)(2-\alpha)}+\frac{2 \alpha}{K(\alpha)(2-\alpha)} \int_{0}^{t} \ell_{4}\left(R(y)-Y_{1, n}(y)\right) \mathrm{d} y, \\
& V(t)-V_{n-1}(t)=\frac{2 \ell_{5}\left(V(t)-Y_{2, n}(t)\right)(1-\alpha)}{K(\alpha)(2-\alpha)}+\frac{2 \alpha}{K(\alpha)(2-\alpha)} \int_{0}^{t} \ell_{5}\left(V(y)-Y_{1, n}(y)\right) \mathrm{d} y .
\end{aligned}
$$

Applying norm on both sides and the Lipschitz axiom,

$$
\begin{aligned}
& \left\|S(t)-\frac{2(1-\alpha) \ell_{1}(S(t), t)}{(2-\alpha) K(\alpha)}-S(0)-\frac{2 \alpha}{(2-\alpha) K(\alpha)} \int_{0}^{t} \ell_{1}(S(y), y) \mathrm{d} y\right\| \\
& \quad \leq\left\|\Upsilon_{1, n}(t)\right\|\left\{1+\left(\frac{2(1-\alpha) \tau_{1}}{(2-\alpha) K(\alpha)}+\frac{2 \alpha \tau_{2} t}{(2-\alpha) K(\alpha)}\right)\right\}, \\
& \left\|A(t)-\frac{2(1-\alpha) \ell_{2}(A(t), t)}{(2-\alpha) K(\alpha)}-A(0)-\frac{2 \alpha}{(2-\alpha) K(\alpha)} \int_{0}^{t} \ell_{2}(A(y), y) \mathrm{d} y\right\| \\
& \quad \leq\left\|\Upsilon_{2, n}(t)\right\|\left\{1+\left(\frac{2(1-\alpha) \tau_{3}}{(2-\alpha) K(\alpha)}+\frac{2 \alpha \tau_{4} t}{(2-\alpha) K(\alpha)}\right)\right\}, \\
& \left\|C(t)-\frac{2(1-\alpha) \ell_{3}(S(t), t)}{(2-\alpha) K(\alpha)}-C(0)-\frac{2 \alpha}{(2-\alpha) K(\alpha)} \int_{0}^{t} \ell_{3}(C(y), y) \mathrm{d} y\right\| \\
& \quad \leq\left\|\Upsilon_{3, n}(t)\right\|\left\{1+\left(\frac{2(1-\alpha) \tau_{5}}{(2-\alpha) K(\alpha)}+\frac{2 \alpha \tau_{6} t}{(2-\alpha) K(\alpha)}\right)\right\}, \\
& \left\|R(t)-\frac{2(1-\alpha) \ell_{4}(R(t), t)}{(2-\alpha) K(\alpha)}-R(0)-\frac{2 \alpha}{(2-\alpha) K(\alpha)} \int_{0}^{t} \ell_{4}(R(y), y) \mathrm{d} y\right\| \\
& \quad \leq\left\|\Upsilon_{4, n}(t)\right\|\left\{1+\left(\frac{2(1-\alpha) \tau_{7}}{(2-\alpha) K(\alpha)}+\frac{2 \alpha \tau_{8} t}{(2-\alpha) K(\alpha)}\right)\right\}, \\
& \quad \leq\left\|\Upsilon_{5, n}(t)\right\|\left\{1+\left(\frac{2(1-\alpha) \tau_{9}}{(2-\alpha) K(\alpha)}+\frac{2 \alpha \tau_{10} t}{(2-\alpha) K(\alpha)}\right)\right\} . \\
& \left\|V(t)-\frac{2(1-\alpha) \ell_{5}(S(t), t)}{(2-\alpha) K(\alpha)}-V(0)-\frac{2 \alpha}{(2-\alpha) K(\alpha)} \int_{0}^{t} \ell_{5}(V(y), y) \mathrm{d} y\right\|
\end{aligned}
$$


Taking $\lim$ as $t$ approaches $\infty$, we get

$$
\begin{aligned}
& S(t)=\frac{2(1-\alpha) \ell_{1}(S(t), t)}{K(\alpha)(2-\alpha)}+\frac{2 \alpha}{K(\alpha)(2-\alpha)} \int_{0}^{t} \ell_{1}(S(y), y) \mathrm{d} y+S(0), \\
& A(t)=\frac{2(1-\alpha) \ell_{2}(A(t), t)}{(2-\alpha) K(\alpha)}+\frac{2 \alpha}{(2-\alpha) K(\alpha)} \int_{0}^{t} \ell_{2}(A(y), y) \mathrm{d} y+A(0), \\
& C(t)=\frac{2(1-\alpha) \ell_{3}(C(t), t)}{(2-\alpha) K(\alpha)}+\frac{2 \alpha}{(2-\alpha) K(\alpha)} \int_{0}^{t} \ell_{3}(C(y), y) \mathrm{d} y+C(0), \\
& R(t)=\frac{2(1-\alpha) \ell_{4}(R(t), t)}{(2-\alpha) K(\alpha)}+\frac{2 \alpha}{(2-\alpha) K(\alpha)} \int_{0}^{t} \ell_{4}(R(y), y) \mathrm{d} y+R(0) \\
& V(t)=\frac{2(1-\alpha) \ell_{5}(V(t), t)}{(2-\alpha) K(\alpha)}+\frac{2 \alpha}{(2-\alpha) K(\alpha)} \int_{0}^{t} \ell_{5}(R(y), y) \mathrm{d} y+V(0)
\end{aligned}
$$

which proves the conclusion that the solution of the proposed epidemiological model as reported by equation (7) exists.
Proof. On the contradiction basis, we assume that $\left(S^{+}(t), A^{+}(t), B^{+}(t), R^{+}(t), V^{+}(t)\right)$ is another solution of model (7); then,

Theorem 3. The proposed epidemiological model described by equation (7) possesess a unique solution.

$$
\begin{aligned}
& S(t)-S^{+}(t)=\frac{2(1-\alpha)\left\{\ell_{1}(S(t), t)-\ell_{1}\left(S^{+}(t), t\right)\right\}}{K(\alpha)(2-\alpha)} \\
& +\frac{2 \alpha}{K(\alpha)(2-\alpha)} \int_{0}^{t}\left\{\ell_{1}(S(y), y)-\ell_{1}\left(S^{+}(y), y\right)\right\} \mathrm{d} y, \\
& A(t)-A^{+}(t)=\frac{2(1-\alpha)\left\{\ell_{2}(S(t), t)-\ell_{2}\left(S^{+}(t), t\right)\right\}}{K(\alpha)(2-\alpha)} \\
& +\frac{2 \alpha}{K(\alpha)(2-\alpha)} \int_{0}^{t}\left\{\ell_{2}(A(y), y)-\ell_{2}\left(A^{+}(y), y\right)\right\} \mathrm{d} y, \\
& C(t)-C^{+}(t)=\frac{2(1-\alpha)\left\{\ell_{3}(S(t), t)-\ell_{3}\left(C^{+}(t), t\right)\right\}}{K(\alpha)(2-\alpha)} \\
& +\frac{2 \alpha}{K(\alpha)(2-\alpha)} \int_{0}^{t}\left\{\ell_{3}(C(y), y)-\ell_{3}\left(C^{+}(y), y\right)\right\} \mathrm{d} y \\
& R(t)-R^{+}(t)=\frac{2(1-\alpha)\left\{\ell_{4}(R(t), t)-\ell_{1}\left(R^{+}(t), t\right)\right\}}{K(\alpha)(2-\alpha)} \\
& +\frac{2 \alpha}{K(\alpha)(2-\alpha)} \int_{0}^{t}\left\{\ell_{4}(R(y), y)-\ell_{4}\left(R^{+}(y), y\right)\right\} \mathrm{d} y, \\
& V(t)-V^{+}(t)=\frac{2(1-\alpha)\left\{\ell_{5}(V(t), t)-\ell_{5}\left(V^{+}(t), t\right)\right\}}{K(\alpha)(2-\alpha)} \\
& +\frac{2 \alpha}{K(\alpha)(2-\alpha)} \int_{0}^{t}\left\{\ell_{5}(V(y), y)-\ell_{5}\left(V^{+}(y), y\right)\right\} \mathrm{d} y .
\end{aligned}
$$


Majorizing the above equations, we obtain

$$
\begin{aligned}
& \left\|S(t)-S^{+}(t)\right\|=\frac{2(1-\alpha)\left\|\ell_{1}(S(t), t)-\ell_{1}\left(S^{+}(t), t\right)\right\|}{K(\alpha)(2-\alpha)} \\
& +\frac{2 \alpha}{K(\alpha)(2-\alpha)} \int_{0}^{t}\left\|\ell_{1}(S(y), y)-\ell_{1}\left(S^{+}(y), y\right)\right\| \mathrm{d} y \\
& \left\|A(t)-A^{+}(t)\right\|=\frac{2(1-\alpha)\left\|\ell_{2}(A(t), t)-\ell_{2}\left(A^{+}(t), t\right)\right\|}{K(\alpha)(2-\alpha)} \\
& +\frac{2 \alpha}{K(\alpha)(2-\alpha)} \int_{0}^{t}\left\|\ell_{2}(A(y), y)-\ell_{2}\left(A^{+}(y), y\right)\right\| \mathrm{d} y, \\
& \left\|C(t)-C^{+}(t)\right\|=\frac{2(1-\alpha)\left\|\ell_{3}(C(t), t)-\ell_{3}\left(C^{+}(t), t\right)\right\|}{K(\alpha)(2-\alpha)} \\
& +\frac{2 \alpha}{K(\alpha)(2-\alpha)} \int_{0}^{t}\left\|\ell_{3}(C(y), y)-\ell_{3}\left(C^{+}(y), y\right)\right\| \mathrm{d} y, \\
& \left\|R(t)-R^{+}(t)\right\|=\frac{2(1-\alpha)\left\|\ell_{4}(R(t), t)-\ell_{4}\left(R^{+}(t), t\right)\right\|}{K(\alpha)(2-\alpha)} \\
& +\frac{2 \alpha}{K(\alpha)(2-\alpha)} \int_{0}^{t}\left\|\ell_{4}(R(y), y)-\ell_{4}\left(R^{+}(y), y\right)\right\| \mathrm{d} y, \\
& \left\|V(t)-V^{+}(t)\right\|=\frac{2(1-\alpha)\left\|\ell_{5}(V(t), t)-\ell_{5}\left(V^{+}(t), t\right)\right\|}{K(\alpha)(2-\alpha)} \\
& +\frac{2 \alpha}{K(\alpha)(2-\alpha)} \int_{0}^{t}\left\|\ell_{5}(V(y), y)-\ell_{5}\left(V^{+}(y), y\right)\right\| \mathrm{d} y .
\end{aligned}
$$

Using Theorems 1 and 2, one may obtain

$$
\begin{gathered}
\left\|S(t)-S^{+}(t)\right\| \leq \frac{2 \tau_{1} \psi_{1}(1-\alpha)}{K(\alpha)(2-\alpha)}+\left(\frac{2 \tau_{2} \alpha \phi_{2} t}{K(\alpha)(2-\alpha)}\right)^{n}, \\
\left\|A(t)-A^{+}(t)\right\| \leq \frac{2 \tau_{3}(1-\alpha) \psi_{3}}{K(\alpha)(2-\alpha)}+\left(\frac{2 \tau_{4} \alpha \phi_{4} t}{K(\alpha)(2-\alpha)}\right)^{n}, \\
\left\|C(t)-C^{+}(t)\right\| \leq \frac{2(1-\alpha) \tau_{5} \psi_{5}}{K(\alpha)(2-\alpha)}+\left(\frac{2 \alpha \tau_{6} \phi_{6} t}{K(\alpha)(2-\alpha)}\right)^{n}, \\
\left\|R(t)-R^{+}(t)\right\| \leq \frac{2 \tau_{7} \psi_{7}(1-\alpha)}{K(\alpha)(2-\alpha)}+\left(\frac{2 \alpha \tau_{8} \phi_{8} t}{K(\alpha)(2-\alpha)}\right)^{n}, \\
\left\|V(t)-V^{+}(t)\right\| \leq \frac{2 \tau_{9} \psi_{9}(1-\alpha)}{K(\alpha)(2-\alpha)}+\left(\frac{2 \alpha \tau_{10} \phi_{10} t}{K(\alpha)(2-\alpha)}\right)^{n} .
\end{gathered}
$$


The inequalities as reported by equation (24) hold for every value of $n$; thus, we obtain

$$
\begin{aligned}
& S(t)=S^{+}(t), \\
& A(t)=A^{+}(t), \\
& B(t)=B^{+}(t), \\
& R(t)=R^{+}(t), \\
& V(t)=V^{+}(t) .
\end{aligned}
$$

We now discuss the positivity as well as the boundedness of model (7) to show the well-posedness of the problem.
Furthermore, we define a certain region for the dynamics of the proposed problem which is positively invariant. For this, the following lemmas have been explored.

Lemma 1. Since $(S(t), A(t), C(t), R(t), V(t))$ are the solutions of model (7), let us consider that the model possesses nonnegative initial conditions; then, the solutions $(S(t), A(t), C(t), R(t), V(t))$ are nonnegative for all $t \geq 0$.

Proof. We assume a general fractional-order $(\omega)$ model of system (7) as

$$
\left\{\begin{aligned}
{ }^{G} D_{0, t}^{\omega}(S(t)) & =\left(1-\zeta^{\alpha}\right) \Pi^{\alpha}-\beta^{\alpha} S(t) A(t)-\rho^{\alpha} \beta^{\alpha} S(t) C(t)-\left(\vartheta^{\alpha}+\eta^{\alpha}\right) S(t)+\sigma^{\alpha} V(t), \\
{ }^{G} D_{0, t}^{\omega}(A(t)) & =p\left\{\beta^{\alpha} S(t) A(t)+\rho^{\alpha} \beta^{\alpha} S(t) C(t)\right\}-\left(\vartheta^{\alpha}+\gamma^{\alpha}\right) A(t), \\
{ }^{G} D_{0, t}^{\omega}(C(t)) & =(1-p)\left\{\beta^{\alpha} S(t) A(t)+\rho^{\alpha} \beta^{\alpha} S(t) C(t)\right\}+q \gamma^{\alpha} A(t)-\left(\vartheta^{\alpha}+\varepsilon^{\alpha}+\tau^{\alpha}\right) C(t), \\
{ }^{G} D_{0, t}^{\omega}(R(t)) & =(1-q) \gamma^{\alpha} A(t)+\eta^{\alpha} S(t)+\tau^{\alpha} C(t)-\vartheta^{\alpha} R(t), \\
{ }^{G} D_{0, t}^{\omega}(V(t)) & =\zeta^{\alpha} \Pi^{\alpha}-\left(\vartheta^{\alpha}+\sigma^{\alpha}\right) V(t),
\end{aligned}\right.
$$

where $G$ represents the fractional-order operator under consideration and $\omega$ is the order. So, equation (26) becomes

$$
\begin{aligned}
& \left.{ }^{G} D_{0, t}^{\omega}(S(t))\right|_{\mathcal{K}(S)}=\left(1-\zeta^{\omega}\right) \Pi^{\omega}>0,\left.{ }^{G} D_{0, t}^{\omega}(A(t))\right|_{\mathcal{K}(A)}=p\left\{\beta^{\omega} S(t) A(t)+\rho^{\omega} \beta^{\omega} S(t) C(t)\right\} \geq 0, \\
& \left.{ }^{G} D_{0, t}^{\omega}(C(t))\right|_{\mathcal{K}(C)}=(1-p)\left\{\beta^{\omega} S(t) A(t)+\rho^{\omega} \beta^{\omega} S(t) C(t)\right\}+q \gamma^{\omega} A(t) \geq 0, \\
& \left.{ }^{G} D_{0, t}^{\omega}(R(t))\right|_{\mathcal{K}(R)}=(1-q) \gamma^{\omega} A(t)+\eta^{\omega} S(t)+\tau^{\omega} C(t)>0,\left.{ }^{G} D_{0, t}^{\omega}(V(t))\right|_{\mathcal{K}(V)}=\zeta^{\alpha} \Pi^{\alpha}>0,
\end{aligned}
$$

where $\kappa(\xi)=\left\{\xi=0\right.$ and $S, A, C, R, V$ contained in $C\left(R_{+} \times\right.$ $\left.\left.R_{+}\right)\right\}$and $\xi \in\{S, A, C, R, V\}$. By following [31], we conclude that the solutions $(S(t), A(t), C(t), R(t), V(t))$ are positive for all nonnegative $t$.

Lemma 2. Let $\Omega$ be the region for dynamics of model (7) within it which is positively invariant; then,

$$
\begin{aligned}
\Omega= & \{(S(t), A(t), C(t), R(t), V(t)) \in \\
& \left.R_{+}^{4}: S+A+C+R+V \leq\left(\frac{\Pi}{\vartheta}\right)^{\omega}\right\} .
\end{aligned}
$$

Proof. Since $N$ represents the total population, therefore, it implies that

$$
{ }^{G} D_{0, t}^{\omega}(T(t))+\vartheta^{\omega} T(t) \leq \Pi^{\omega}
$$

The solution of equation (29) gives

$$
T(t) \leq T(0) E_{\omega}\left(-\vartheta^{\omega} t^{\omega}\right)+\left(\frac{\Pi}{\vartheta}\right)^{\omega}\left(1-E_{\omega}\left(-\vartheta^{\omega} t^{\omega}\right)\right)
$$

where $E($.$) is the Mittag-Leffler function such that$ $E_{\omega}(Z)=\sum_{n=0}^{\infty} Z^{n} / \Gamma(\omega i+1)$. Note that, in equation (30), whenever time increases with no bound, $T(t) \longrightarrow(\Pi / \vartheta)^{\omega}$. Hence, if $T(0) \leq(\Pi / \vartheta)^{\omega}$, then $T(t) \leq(\Pi / \vartheta)^{\omega}$ for all $t>0$, while if $T(0)>(\Pi / \vartheta)^{\omega}$, then $T$ goes into the feasible region $\Omega$ and will never leave. So, it could be concluded that the dynamics of the fractional-order epidemiological model can be studied in the feasible region $\Omega$.

\section{Steady-State Analysis}

The proposed epidemiological model (7) of the hepatitis B virus is examined for the equilibria: disease-free and endemic states. Let $D_{1}$ be the disease-free equilibrium of the proposed model; then, for analyzing this point, the population under consideration is assumed to be infection free. Thus, the system reported by equation (7) has a disease-free equilibrium $D_{1}=\left(S^{0}, A^{0}, C^{0}, R^{0}, V^{0}\right)$, where $S^{0}=$ $q_{4}^{\alpha}\left(1-\zeta^{\alpha}\right)+\sigma^{\alpha} \zeta^{\alpha} / q_{1}^{\alpha} q_{4}^{\alpha}, A^{0}=C^{0}=0, R^{0}=\eta^{\alpha} \Pi^{\alpha} q_{4}^{\alpha}\left(1-\zeta^{\alpha}\right)+$ $\sigma^{\alpha} \zeta^{\alpha} / \vartheta^{\alpha} q_{1}^{\alpha} q_{4}^{\alpha}$, and $V^{0}=\zeta^{\alpha} \Pi^{\alpha} / q_{4}^{\alpha}$, and $q_{1}=\vartheta^{\alpha}+\eta^{\alpha}$, $q_{2}=\vartheta^{\alpha}+\gamma^{\alpha}, q_{3}=\vartheta^{\alpha}+\varepsilon^{\alpha}+\tau^{\alpha}$, and $q_{4}=\vartheta^{\alpha}+\sigma^{\alpha}$. Now, to calculate the basic reproductive number, we assume $X=(A, C)^{T}$; then, system $(7)$ yields 


$$
\left.\frac{\mathrm{d} X}{\mathrm{~d} t}\right|_{D_{1}}=F-V
$$

where

$$
\begin{aligned}
& F=\left[\begin{array}{cc}
p \beta^{\alpha} S^{0} & p \rho^{\alpha} \beta^{\alpha} S^{0} \\
(1-p) \beta^{\alpha} S^{0} & (1-p) \rho^{\alpha} \beta^{\alpha} S^{0}
\end{array}\right], \\
& V=\left[\begin{array}{cc}
q_{2} & 0 \\
-q \gamma^{\alpha} & q_{3}
\end{array}\right] .
\end{aligned}
$$

Therefore, the basic reproductive number is the spectral radius of $\rho\left(F V^{-1}\right)$, i.e., $R_{0}=R_{1}+R_{2}+R_{3}$, where

$$
\begin{aligned}
& R_{1}=\frac{p \beta^{\alpha} S^{0}}{q_{2}}, \\
& R_{2}=\frac{\rho^{\alpha} \beta^{\alpha} S^{0}(1-p)}{q_{3}}, \\
& R_{3}=\frac{p \gamma^{\alpha} \rho^{\alpha} \beta^{\alpha} q S^{0}}{q_{2} q_{3}} .
\end{aligned}
$$

Let $D_{2}$ be the endemic equilibrium, and assume that $S=S^{*}, A=A^{*}, C=C^{*}, R=R^{*}$, and $V=V^{*}$ at the steady state of the proposed model; then, the solution of the resultant algebraic equations will lead to the endemic equilibrium. Thus, regarding the local as well as global analysis of the proposed model, we have the following stability results.

Theorem 4. If $R_{0}<1$, then the disease-free equilibrium $D_{1}$ of the proposed model (7) is locally asymptotically stable, while if $R_{0}>1$, then the endemic equilibrium $D_{2}$ is locally asymptotically stable.

Proof. The linearizable version of the proposed hepatitis B model (7) around $D_{1}$ leads to a matrix given by

$$
\left.J\right|_{D_{1}}=\left(\begin{array}{ccccc}
-q_{1} & -\beta^{\alpha} S^{0} & -\rho^{\alpha} \beta^{\alpha} S^{0} & 0 & \sigma^{\alpha} \\
0 & p \beta^{\alpha} S^{0}-q_{2} & p \rho^{\alpha} \beta^{\alpha} S^{0} & 0 & 0 \\
0 & (1-p) \beta^{\alpha} S^{0}+q \gamma & (1-p) \rho^{\alpha} \beta^{\alpha} S^{0}-q_{3} & 0 & 0 \\
\eta^{\alpha} & (1-q) \gamma^{\alpha} & \tau^{\alpha} & -\vartheta^{\alpha} & 0 \\
0 & 0 & 0 & 0 & -q_{4}
\end{array}\right) .
$$

The characteristic equation of the matrix $\left.J\right|_{D_{1}}$ takes the following form:

$$
\omega^{5}+a_{1} \omega^{4}+a_{2} \omega^{3}+a_{3} \omega^{2}+a_{4} \omega+a_{5},
$$

where

$$
\begin{aligned}
a_{1}= & q_{1}+q_{4}+\vartheta^{\alpha}+q_{2}\left(1-R_{1}\right)+q_{3}\left(1-R_{2}\right), \\
a_{2}= & q_{1} q_{4}+\left(q_{1}+q_{4}\right)\left\{\vartheta^{\alpha}+q_{2}\left(1-R_{1}\right)+q_{3}\left(1-R_{2}\right)\right\} \\
& +q_{2} q_{3}\left(1-R_{0}\right)+q_{3} \vartheta^{\alpha}\left(1-R_{2}\right)+q_{2} \vartheta^{\alpha}\left(1-R_{1}\right), \\
a_{3}= & \left(q_{1}+q_{4}\right)\left\{q_{2} \vartheta^{\alpha}\left(1-R_{1}\right)+q_{3} \vartheta^{\alpha}\left(1-R_{2}\right)+q_{2} q_{3}\left(1-R_{0}\right)\right\} \\
& +q_{1} q_{4}\left\{\vartheta^{\alpha}+q_{2}\left(1-R_{1}\right)+q_{3}\left(1-R_{2}\right)\right\}+q_{2} q_{3} \vartheta^{\alpha}\left(1-R_{0}\right), \\
a_{4}= & q_{1} q_{4}\left\{q_{2} q_{3}\left(1-R_{0}\right)+q_{2} \vartheta^{\alpha}\left(1-R_{1}\right)+q_{3} \vartheta^{\alpha}\left(1-R_{2}\right)\right\}+\left(q_{1}+q_{4}\right) q_{2} q_{3} \vartheta^{\alpha}\left(1-R_{0}\right), \\
a_{5}= & q_{1} q_{4} q_{2} q_{3} \vartheta^{\alpha}\left(1-R_{0}\right) .
\end{aligned}
$$

It could be noted that the real parts of the eigenvalues of the above matrix $\left.J\right|_{D_{1}}$ are negative whenever Routh-Hurwitz criteria, i.e., $\quad H:\left\{a_{i}>0\right.$, for $i=1,2,3,4,5, a_{1} a_{2} a_{3}-$ $a_{3}^{2}-a_{1}^{2} a_{4}>0$ and $\left(a_{1} a_{4}-a_{5}\right)\left(a_{1} a_{2} a_{3}-a_{3}^{2}-a_{1}^{2} a_{4}\right)-a_{5}\left(a_{1} a_{2}\right.$ $\left.\left.-a_{3}\right)^{2}-a_{1} a_{5}^{2}>0\right\}$, hold. So,

$$
\begin{aligned}
a_{1} a_{2} a_{3}-a_{3}^{2}-a_{1}^{2} a_{4}= & \left\{q_{1}+q_{4}+\vartheta^{\alpha}+q_{2}\left(1-R_{1}\right)+q_{3}\left(1-R_{2}\right)\right\}\left\{q_{1} q_{4}+\left(q_{1}+q_{4}\right)\left\{\vartheta^{\alpha}+q_{2}\left(1-R_{1}\right)\right.\right. \\
& \left.\left.+q_{3}\left(1-R_{2}\right)\right\}+q_{2} q_{3}\left(1-R_{0}\right)+q_{3} \vartheta^{\alpha}\left(1-R_{2}\right)+q_{2} \vartheta^{\alpha}\left(1-R_{1}\right)\right\}\left\{( q _ { 1 } + q _ { 4 } ) \left\{q_{2} \vartheta^{\alpha}\left(1-R_{1}\right)\right.\right. \\
& \left.+q_{3} \vartheta^{\alpha}\left(1-R_{2}\right)+q_{2} q_{3}\left(1-R_{0}\right)\right\}+q_{1} q_{4}\left(\vartheta^{\alpha}+q_{2}\left(1-R_{1}\right)+q_{3}\left(1-R_{2}\right)\right) \\
& \left.+q_{2} q_{3} \vartheta^{\alpha}\left(1-R_{0}\right)\right\}-\left\{\left(q_{1}+q_{4}\right)\left\{q_{2} \vartheta^{\alpha}\left(1-R_{1}\right)+q_{3} \vartheta^{\alpha}\left(1-R_{2}\right)+q_{2} q_{3}\left(1-R_{0}\right)\right\}\right. \\
& \left.+q_{1} q_{4}\left\{\vartheta^{\alpha}+q_{2}\left(1-R_{1}\right)+q_{3}\left(1-R_{2}\right)\right\}+q_{2} q_{3} \vartheta^{\alpha}\left(1-R_{0}\right)\right\}^{2}-\left(q_{1}+q_{4}+\vartheta^{\alpha}\right. \\
& \left.+q_{2}\left(1-R_{1}\right)+q_{3}\left(1-R_{2}\right)\right)^{2}\left\{q_{1} q_{4}\left(q_{2}\left(1-R_{1}\right)+q_{3}\left(1-R_{2}\right)+q_{2} q_{3}\left(1-R_{0}\right)\right)+\left(q_{1}+q_{4}\right) q_{2} q_{3} \vartheta^{\alpha}\left(1-R_{0}\right)\right\},
\end{aligned}
$$


and

$$
\begin{aligned}
& \left(a_{1} a_{4}-a_{5}\right)\left(a_{1} a_{2} a_{3}-a_{3}^{2}-a_{1}^{2} a_{4}\right)-a_{5}\left(a_{1} a_{2}-a_{3}\right)^{2}-a_{1} a_{5}^{2} \\
& \left(q_{1}+q_{4}+\vartheta^{\alpha}+q_{2}\left(1-R_{1}\right)+q_{3}\left(1-R_{2}\right)\right) \\
& \left\{\begin{array}{c}
\left(q_{1} q_{4}\left(q_{2} \vartheta^{\alpha}\left(1-R_{1}\right)+q_{3} \vartheta^{\alpha}\left(1-R_{2}\right)+q_{2} q_{3}\left(1-R_{0}\right)\right)+\left(q_{1}+q_{4}\right) q_{2} q_{3}\left(1-R_{0}\right)+\left(q_{1}+q_{4}\right) q_{2} q_{3} \vartheta^{\alpha}\left(1-R_{0}\right)\right) \\
-q_{1} q_{4} q_{2} q_{3} \vartheta^{\alpha}\left(1-R_{0}\right)
\end{array}\right\} \\
& \left\{\begin{array}{c}
\left(q_{1}+q_{4}+\vartheta^{\alpha}+q_{2}\left(1-R_{1}\right)+q_{3}\left(1-R_{2}\right)\right)\left\{q_{1} q_{4}+\left(q_{1}+q_{4}\right)\left\{\vartheta^{\alpha}+q_{2}\left(1-R_{1}\right)+q_{3}\left(1-R_{2}\right)\right\}+q_{2} q_{3}\left(1-R_{0}\right)+q_{3} \vartheta^{\alpha}\left(1-R_{2}\right)+q_{2} \vartheta^{\alpha}\left(1-R_{1}\right)\right\} \\
\left\{\left(q_{1}+q_{4}\right)\left\{q_{2} \vartheta^{\alpha}\left(1-R_{1}\right)+q_{3} \vartheta^{\alpha}\left(1-R_{2}\right)+q_{2} q_{3}\left(1-R_{0}\right)\right\}+q_{1} q_{4}\left\{\vartheta^{\alpha}+q_{2}\left(1-R_{1}\right)+q_{3}\left(1-R_{2}\right)\right\}+q_{2} q_{3} \vartheta^{\alpha}\left(1-R_{0}\right)\right\} \\
-\left\{\left(q_{1}+q_{4}\right)\left\{q_{2} \vartheta^{\alpha}\left(1-R_{1}\right)+q_{3} \vartheta^{\alpha}\left(1-R_{2}\right)+q_{2} q_{3}\left(1-R_{0}\right)\right\}+q_{1} q_{4}\left(\vartheta^{\alpha}+q_{2}\left(1-R_{1}\right)+q_{3}\left(1-R_{2}\right)\right)+q_{2} q_{3} \vartheta\left(1-R_{0}\right)\right\}^{2} \\
-\left\{q_{1}+q_{4}+\vartheta+q_{2}\left(1-R_{1}\right)+q_{3}\left(1-R_{2}\right)\right\}^{2}\left(q_{1} q_{4}\left(q_{2} \vartheta^{\alpha}\left(1-R_{1}\right)+q_{3} \vartheta^{\alpha}\left(1-R_{2}\right)+q_{2} q_{3}\left(1-R_{0}\right)\right)+\left(q_{1}+q_{4}\right) q_{2} q_{3} \vartheta^{\alpha}\left(1-q_{2}\left(1-R_{1}\right)+q_{3}\left(1-R_{2}\right)\right)\right.
\end{array}\right\} \\
& \left\{\begin{array}{c}
\left\{q_{1} q_{4}+\left(q_{1}+q_{4}\right)\left\{\vartheta^{\alpha}+q_{2}\left(1-R_{1}\right)+q_{3}\left(1-R_{2}\right)\right\}+q_{2} q_{3}\left(1-R_{0}\right)+q_{3} \vartheta^{\alpha}\left(1-R_{2}\right)+q_{2} \vartheta^{\alpha}\left(1-R_{1}\right)\right\} \\
-\left(q_{1}+q_{4}\right)\left\{q_{2} \vartheta^{\alpha}\left(1-R_{1}\right)+q_{3} \vartheta^{\alpha}\left(1-R_{2}\right)+q_{2} q_{3}\left(1-R_{0}\right)\right\}-q_{1} q_{4}\left(\vartheta^{\alpha}+q_{2} \vartheta^{\alpha}\left(1-R_{1}\right)+q_{3} \vartheta^{\alpha}\left(1-R_{2}\right)\right)
\end{array}\right. \\
& -q_{1} q_{2} q_{3} q_{4} \vartheta^{\alpha}\left(1-R_{0}\right)\left\{\begin{array}{c}
2 \\
-q_{2} q_{3} \vartheta^{\alpha}\left(1-R_{0}\right)
\end{array}\right.
\end{aligned}
$$$$
-\left(q_{1}+q_{4}+\vartheta^{\alpha}+q_{2}\left(1-R_{1}\right)+q_{3}\left(1-R_{2}\right)\right) q_{1}^{2} q_{4}^{2} q_{2}^{2} q_{3}^{2} \vartheta^{2 \alpha}\left(1-R_{0}\right)^{2} .
$$

Clearly, we observe that all the coefficients $a_{i}$ for $i=$ $1,2,3,4,5$ are positive whenever $R_{0}<1$, and if $a_{1} a_{2} a_{3}-a_{3}^{2}-$ $a_{1}^{2} a_{4}$ and $\left(a_{1} a_{4}-a_{5}\right)\left(a_{1} a_{2} a_{3}-a_{3}^{2}-a_{1}^{2} a_{4}\right)-a_{5}\left(a_{1} a_{2}-a_{3}\right)^{2}-$ $a_{1} a_{5}^{2}$ are positive, then it implies that the Routh-Hurwitz criteria hold, and so, the disease-free state $D_{1}$ is stable. In a similar fashion, it can proved that the disease endemic state $D_{2}$ of the proposed model (7) is stable.

Theorem 5. If $R_{0} \leq 1$, then the disease-free equilibrium $D_{1}$ of the proposed model (7) is globally asymptotically stable, while if $R_{0}>1$, then the endemic equilibrium $D_{2}$ is globally asymptotically stable.

Proof. Let $\chi(t)=(S(t), A(t), C(t), R(t), V(t))$, and we claim that it has a finite limit whenever $t$ approaches to $\infty$; then, the last equation of model (7) looks like

$$
{ }^{C F} D_{0, t}^{\alpha} V(t)=\zeta^{\alpha} \Pi^{\alpha}-q_{4} V(t)
$$

Since for $t \geq 0$ and for any $\varphi, \varphi \leq \varphi e^{t}$, by following the mean value theorem and the result as stated by Theorem 3.1 in [32], equation (39) implies that

$$
\|V(t)\| \leq a C \exp ^{\left[-\left\{q_{4}\right\}^{1 / \alpha}+1\right] t}
$$

where $a=\left\|V_{0}\right\| e^{-T}+K T^{\alpha} e^{-T} / \alpha \Gamma(\alpha)+\zeta^{\alpha} \Pi^{\alpha}, t \geq T$, and $C$ is a positive constant, and consequently, we obtain

$$
\lim _{t \rightarrow \infty} V(t) \leq C(\zeta \Pi)^{\alpha} \text {. }
$$

Similarly, the first equation of the proposed fractionalorder model (7) can be rewritten as

$$
{ }^{C F} D_{0, t}^{\alpha} S(t) \leq\left(1-\zeta^{\alpha}\right) \Pi^{\alpha}-q_{1} S(t)+\sigma^{\alpha} V(t) .
$$
then,

Let $b=\left\|S_{0}\right\| e^{-T}+K T^{\alpha} e^{-T} / \alpha \Gamma(\alpha)+\left(1-\zeta^{\alpha}\right) \Pi^{\alpha}+\sigma^{\alpha V(t)}$;

$$
\|S(t)\| \leq b C \exp ^{\left[-\left\{q_{1}\right\}^{1 / \alpha}+1\right] t},
$$

which implies that

$$
\lim _{t \longrightarrow \infty} S(t) \leq C\left(\left(1-\zeta^{\alpha}\right) \Pi^{\alpha}\right)+\lim _{t \longrightarrow \infty} \sigma^{\alpha} V(t),
$$

or equivalently, equation (44) may take the form after using equation (41) in equation (44) such that

$$
\lim _{t \rightarrow \infty} S(t) \leq C \Pi^{\alpha} \text {. }
$$

In a similar fashion, lim of $A(t), C(t)$, and $R(t)$ can be shown. Moreover, we assume that

$$
\lim _{t \longrightarrow \infty} \chi(t)=\left(S_{\infty}, A_{\infty}, C_{\infty}, R_{\infty}, V_{\infty}\right),
$$


and

$$
\begin{aligned}
\phi(\chi) & =\left(\begin{array}{l}
\phi_{1}(\chi) \\
\phi_{2}(\chi) \\
\phi_{3}(\chi) \\
\phi_{4}(\chi) \\
\phi_{5}(\chi)
\end{array}\right) \\
& =\left(\begin{array}{c}
\left(1-\zeta^{\alpha}\right) \Pi^{\alpha}-\beta^{\alpha} S(t) A(t)-\rho^{\alpha} \beta^{\alpha} S(t) C(t)-q_{1} S(t)+\sigma^{\alpha} V(t) \\
p\left\{\beta^{\alpha} S(t) A(t)+\rho^{\alpha} \beta^{\alpha} S(t) C(t)\right\}-q_{2} A(t) \\
(1-p)\left\{\beta^{\alpha} S(t) A(t)+\rho^{\alpha} \beta^{\alpha} S(t) C(t)\right\}+q \gamma^{\alpha} A(t)-q_{3} C(t) \\
(1-q) \gamma^{\alpha} A(t)+\eta^{\alpha} S(t)+\tau^{\alpha} C(t)-\vartheta^{\alpha} R(t) \\
\zeta^{\alpha} \Pi^{\alpha}-q_{4} V(t)
\end{array}\right) .
\end{aligned}
$$

Thus, in light of the mean value theorem, there exist positive constants $C_{1}$ and $C_{2}$ such that

$$
\|\phi(\chi)\| \leq C_{1}+C_{2}\|\chi\| .
$$

So, Theorems 2.1 and 3.1 in [33] imply that ${ }^{C F} D_{0, t}^{\alpha}(S(t), A(t), C(t), R(t), V(t))$ is uniformly continuous. Thus, Barbalat's lemma (for details, see [34]) implies that

$$
\underset{t \longrightarrow \infty}{\operatorname{Cim}} D_{0, t}^{\alpha}(\chi(t))=(0,0,0,0,0) .
$$

Consequently,

$$
\left\{\begin{array}{l}
\left(1-\zeta^{\alpha}\right) \Pi^{\alpha}-\beta^{\alpha} S(t) A(t)-\rho^{\alpha} \beta^{\alpha} S(t) C(t)-q_{1} S(t)+\sigma^{\alpha} V(t)=0 \\
p\left\{\beta^{\alpha} S(t) A(t)+\rho^{\alpha} \beta^{\alpha} S(t) C(t)\right\}-q_{2} A(t)=0 \\
(1-p)\left\{\beta^{\alpha} S(t) A(t)+\rho^{\alpha} \beta^{\alpha} S(t) C(t)\right\}+q \gamma^{\alpha} A(t)-q_{3} C(t)=0 \\
(1-q) \gamma^{\alpha} A(t)+\eta^{\alpha} S(t)+\tau^{\alpha} C(t)-\vartheta^{\alpha} R(t)=0 \\
\zeta^{\alpha} \Pi^{\alpha}-q_{4} V(t)=0 .
\end{array}\right.
$$

Therefore, $\left(S_{\infty}, A_{\infty}, C_{\infty}, R_{\infty}, V_{\infty}\right)$ is an equilibrium point of the proposed fractional-order epidemiological model (7), and by a similar argument as stated by Theorem 3.1 in [35], we conclude that

$$
\begin{aligned}
& \lim _{t \longrightarrow \infty}(\chi(t))=D_{1}, \\
& \lim _{t \longrightarrow \infty}(\chi(t))=D_{2} .
\end{aligned}
$$

Hence, the disease endemic state $D_{2}$ does not exist whenever $R_{0}<1$, and so, $\lim \chi(t)=D_{1}$ as $t$ approaches $\infty$, and if $R_{0}=1$, then $D_{2}=D_{1}$ and $\lim \chi(t)=D_{1}$ as $t$ approaches $\infty$, while on the contrary, if $R_{0}>1$, then $D_{2}$ exists, and thus, $\lim \chi(t)=D_{2}$ as $t$ tends to $\infty$.

\section{Numerical Simulation}

In this section, the numerical simulations are carried out to understand the temporal dynamical behavior corresponding with hepatitis B virus fractional-order epidemiological model (7). This is very important to show the feasibility of the reported work and investigate the validity of the analytical work using large-scale numerical simulation. It is important to point out that, unlike traditional numerical analysis, there are not as many options to choose schemes for the numerical analysis of the fractional-order epidemiological model simulations [36]. Thus, there is a need of extensive research in order to develop new schemes and techniques that are both convergent and robust in the field of 


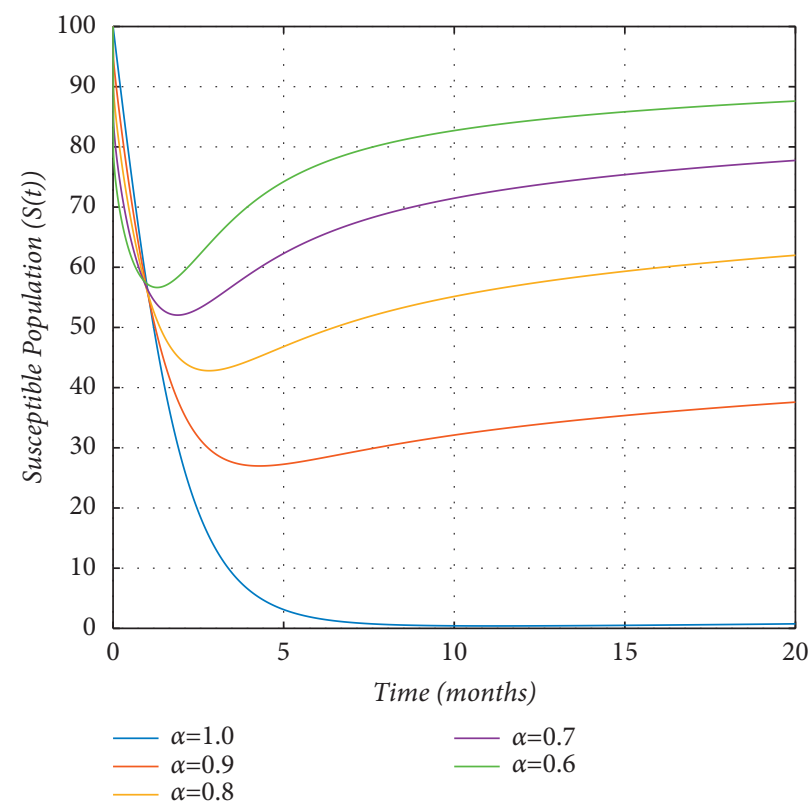

FIgURE 1: The graph shows the dynamics of the susceptible population $(S(t))$ for different values of the fractional-order parameter $(\alpha)$, and the initial population sizes are $(100,90,80,70,60)$.

fractional calculus. By following the numerical schemes as reported in $[37,38]$, we assume $[0, t]$ interval of simulation and $h=10^{-3}$ is the time step for integration, and $n=T / h$, $n \in \mathbb{N}$, and $u=0,1,2, \ldots, n$. So, the scheme may take the following structure:

$$
\begin{aligned}
{ }^{C F} S_{u+1}= & S(0)+(1-\alpha)\left\{\left(1-\zeta^{\alpha}\right) \Pi^{\alpha}-\beta^{\alpha} S(t) A(t)-\rho^{\alpha} \beta^{\alpha} S(t) C(t)-\left(\vartheta^{\alpha}+\eta^{\alpha}\right) S(t)+\sigma^{\alpha} V(t)\right\} \\
& +\alpha h \sum_{k=0}^{u}\left\{\left(1-\zeta^{\alpha}\right) \Pi^{\alpha}-\beta^{\alpha} S(t) A(t)-\rho^{\alpha} \beta^{\alpha} S(t) C(t)-\left(\vartheta^{\alpha}+\eta^{\alpha}\right) S(t)+\sigma^{\alpha} V(t)\right\}, \\
{ }^{C F} A_{u+1}= & A(0)+(1-\alpha)\left\{p\left\{\beta^{\alpha} S(t) A(t)+\rho^{\alpha} \beta^{\alpha} S(t) C(t)\right\}-\left(\vartheta^{\alpha}+\gamma^{\alpha}\right) A(t)\right\} \\
& +\alpha h \sum_{k=0}^{u}\left\{p\left\{\beta^{\alpha} S(t) A(t)+\rho^{\alpha} \beta^{\alpha} S(t) C(t)\right\}-\left(\vartheta^{\alpha}+\gamma^{\alpha}\right) A(t)\right\}, \\
{ }^{C F} C_{u+1}= & C(0)+(1-\alpha)\left\{(1-p)\left\{\beta^{\alpha} S(t) A(t)+\rho^{\alpha} \beta^{\alpha} S(t) C(t)\right\}+q \gamma^{\alpha} A(t)-\left(\vartheta^{\alpha}+\varepsilon^{\alpha}+\tau^{\alpha}\right) C(t)\right\} \\
& +\alpha h \sum_{k=0}^{u}\left\{(1-p)\left\{\beta^{\alpha} S(t) A(t)+\rho^{\alpha} \beta^{\alpha} S(t) C(t)\right\}+q \gamma^{\alpha} A(t)-\left(\vartheta^{\alpha}+\varepsilon^{\alpha}+\tau^{\alpha}\right) C(t)\right\}, \\
{ }^{C F} R_{u+1}= & (1-\alpha)\left\{(1-q) \gamma^{\alpha} A(t)+\eta^{\alpha} S(t)+\tau^{\alpha} C(t)-\vartheta^{\alpha} R(t)\right\} \\
& +\alpha h \sum_{k=0}^{u}\left\{(1-q) \gamma^{\alpha} A(t)+\eta^{\alpha} S(t)+\tau^{\alpha} C(t)-\vartheta^{\alpha} R(t)\right\}+R(0), \\
& +\alpha h \sum_{k=0}^{u}\left\{\zeta^{\alpha} \Pi^{\alpha}-\left(\vartheta^{\alpha}+\sigma^{\alpha}\right) V(t)\right\} . \\
{ }^{C F} V_{u+1}= & (1-\alpha)\left\{\zeta^{\alpha} \Pi^{\alpha}-\left(\vartheta^{\alpha}+\sigma^{\alpha}\right) V(t)\right\}+V(0) \\
&
\end{aligned}
$$

Furthermore, the parameters' value is assumed with biological feasibility; that is, $\zeta=0.4, \quad \Pi=0.0975$, $\vartheta=0.00000456, \varepsilon=0.3454, \beta=0.022, \rho=0.048, \quad p=0.5$, $q=0.5, \gamma=0.45, \eta=0.8613, \tau=0.1428$, and $\sigma=0.06$, and 


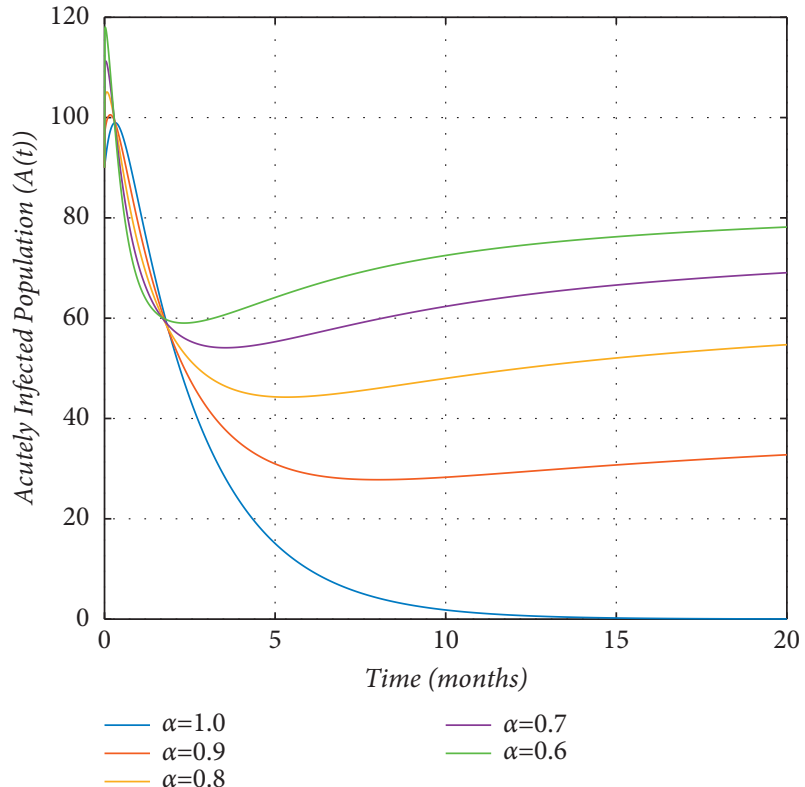

FIGURE 2: The graph visualizes the dynamics of the acutely infected population $(A(t))$ for different values of the fractional-order parameter $(\alpha)$, and the initial population sizes are $(100,90,80,70,60)$.

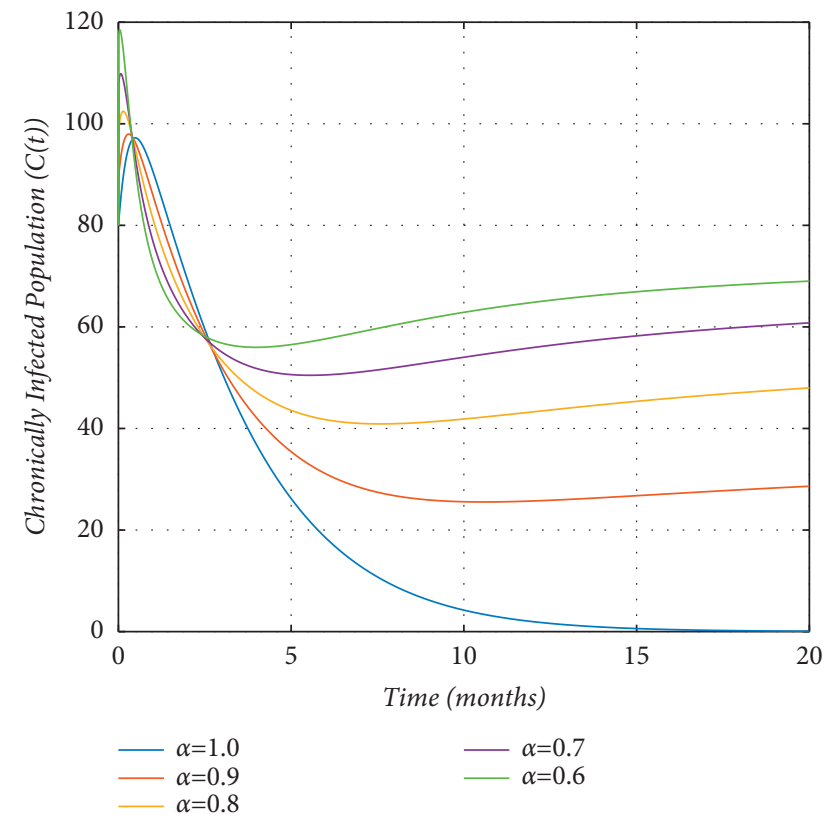

Figure 3: The graph demonstrates the dynamics of the chronically infected population against different values of the fractional-order parameter $(\alpha)$, and the initial sizes of the population are $(100,90,80,70,60)$.

the initial sizes of compartmental populations are $(100,90,80,70,60)$.

By the execution of the above scheme with the stated parameters' value as above along the initial sizes of populations, we obtain the results as depicted in Figures 1-5. These graphs visualize the dynamical behaviors of the

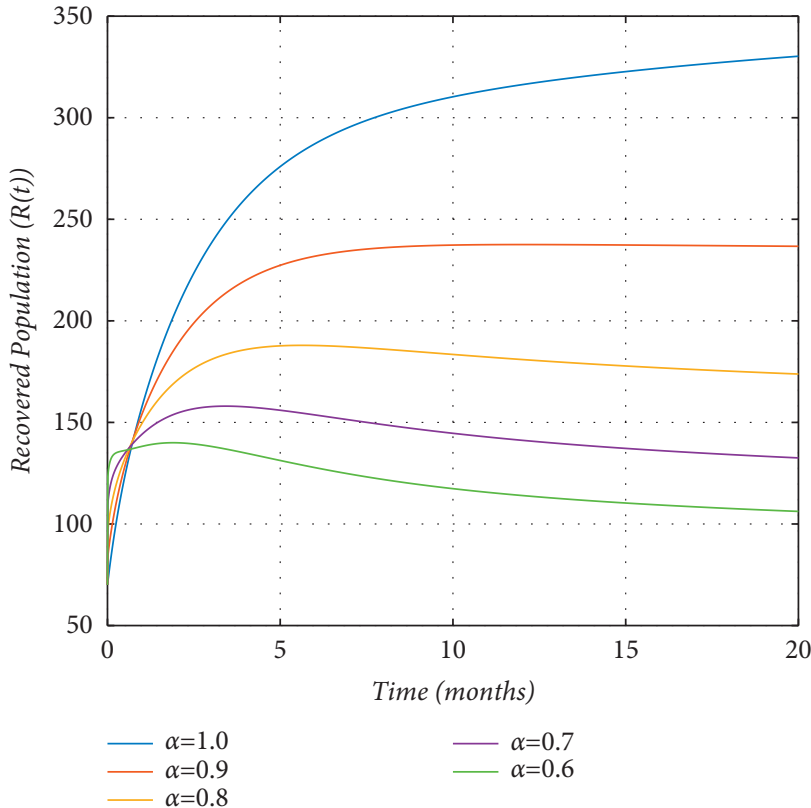

Figure 4: The graph demonstrates the dynamics of the recovered population against different values of the fractional-order parameter $(\alpha)$, and the initial sizes of the population are $(100,90,80,70,60)$.

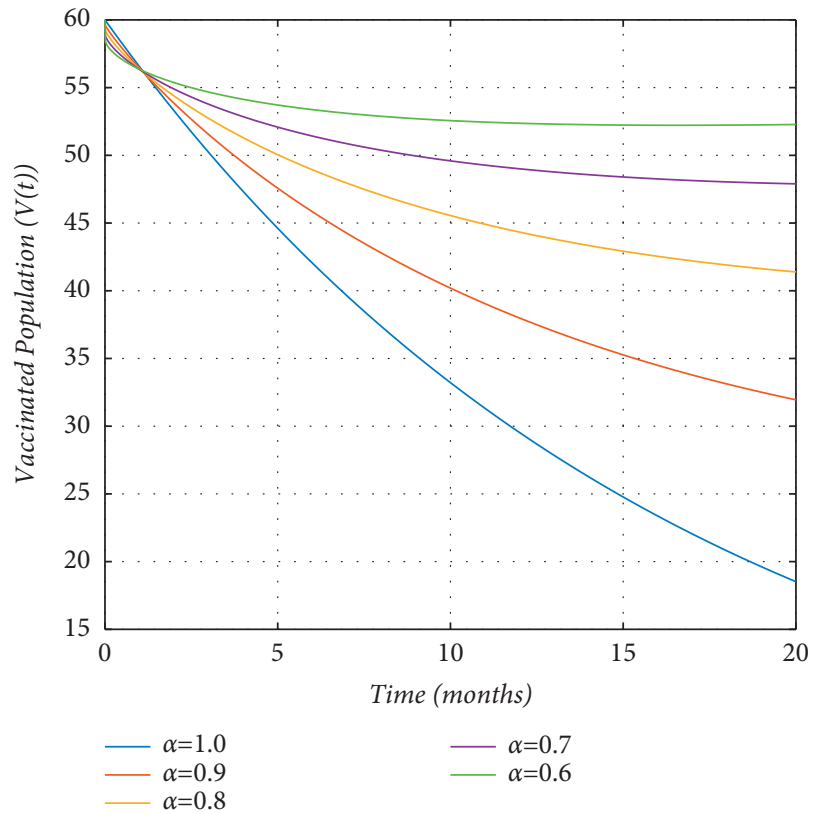

FIGURE 5: The graph demonstrates the dynamics of the vaccinated population against different values of the fractional-order parameter $(\alpha)$, and the initial sizes of the population are $(100,90,80,70,60)$.

susceptible, the acutely and chronically infected, the recovered, and the vaccinated groups of populations. More precisely, the dynamics of the susceptible individuals for different values of the fractional-order parameter $(\alpha)$ is shown in Figure 1, which demonstrates that if the value of $\alpha$ increases, then the ratio of the susceptible individuals 
decreases. This shows that the fractional-order parameter and the susceptible population are inversely proportional to each other. Similarly, the acutely and chronically infected population are also inversely proportional to the fractionalorder parameter $(\alpha)$ as shown in Figures 2 and 3, respectively, while the dynamics of recovered individuals reveals that there is a direct relation between the fractional-order parameter $(\alpha)$ and the recovered population, i.e., whenever the value of $\alpha$ increases, the size of the recovered population also increases as depicted in Figure 4. The dynamics of the vaccinated group of population is described in Figure 5, which demonstrates that the fractional-order parameter $(\alpha)$ has a negative impact on the dynamics of the vaccinated population, i.e., whenever the value of $\alpha$ increases, the size of the population group $V(t)$ decreases. This analysis reveals that the CF fractional-order model presents more valuable outputs regarding the behavior of compartmental populations which usually could not be obtained in case of the classical model.

\section{Conclusion}

The work carried out in this study consists of a new epidemiological model related to dynamics of hepatitis $B$ virus transmission. We used the CF operator and investigated the dynamics of hepatitis B virus. We formulated the proposed model first and then fractionalized by using the Caputo-Fabrizio operator with dimensional balance in respect of involved epidemic parameters. We used the fixed point theory and rigorously showed that the model under the CF operator possesses a unique solution. We also discussed biological as well as mathematical feasibility of the proposed model by proving that the solutions of the model are bounded and positive. Moreover, the basic reproductive number is calculated, and the stability analysis of the steady states of the proposed fractional-order epidemiological model is shown. At the end, we presented some numerical simulations to show the relation between compartmental populations and the fractional-order operator. Thus, the major findings of this study show that the CF fractionalorder operator is the best choice instead of the classical order.

\section{Data Availability}

No data were used to support this study.

\section{Conflicts of Interest}

The authors declare that they have no conflicts of interest.

\section{Acknowledgments}

This research work was funded by the Deanship of Scientific Research, King Khalid University (Project no. RGP.2/204/ 42), Abha, K. S. A. The author, Basem Al Alwan, therefore, acknowledges with thanks to the DSR and the Chemical Engineering Department in the College of Engineering (KKU) for the financial and technical support. The authors would also like to acknowledge and express their gratitude to the United Arab Emirates University, Al Ain, UAE, for providing financial support (Grant no. 12S086).

\section{References}

[1] J. Mann and M. Roberts, "Modelling the epidemiology of hepatitis B in New Zealand," Journal of Theoretical Biology, vol. 269, no. 1, pp. 266-272, 2011.

[2] D. Lavanchy, "Hepatitis B virus epidemiology, disease burden, treatment, and current and emerging prevention and control measures," Journal of Viral Hepatitis, vol. 11, no. 2, pp. 97-107, 2004.

[3] A. S. Lok, E. J. Heathcote, and J. H. Hoofnagle, "Management of hepatitis B: 2000-Summary of a workshop," Gastroenterology, vol. 120, no. 7, pp. 1828-1853, 2001.

[4] B. J. McMahon, "Epidemiology and natural history of hepatitis B," Seminars in Liver Disease, vol. 25, no. 1, pp. 3-8, 2005.

[5] M.-H. Chang, "Hepatitis B virus infection," Seminars in Fetal and Neonatal Medicine, vol. 12, no. 3, pp. 160-167, 2007.

[6] S. Thornley, C. Bullen, and M. Roberts, "Hepatitis B in a high prevalence New Zealand population: a mathematical model applied to infection control policy," Journal of Theoretical Biology, vol. 254, no. 3, pp. 599-603, 2008.

[7] M. K. Libbus and L. M. Phillips, "Public health management of perinatal hepatitis B virus," Public Health Nursing, vol. 26, no. 4, pp. 353-361, 2009.

[8] R. Williams, "Global challenges in liver disease," Hepatology, vol. 44, no. 3, pp. 521-526, 2006.

[9] J. E. Maynard, M. A. Kane, and S. C. Hadler, "Global control of hepatitis B through vaccination: role of hepatitis $B$ vaccine in the expanded programme on immunization," Clinical Infectious Diseases, vol. 11, no. 3, pp. S574-S578, 1989.

[10] C. W. Shepard, E. Simard, L. Finelli, A. Fiore, and B. Bell, "Hepatitis B virus infection: epidemiology and vaccination," Epidemiologic Reviews, vol. 28, no. 1, pp. 112-125, 2006.

[11] K. Wang, A. Fan, and A. Torres, "Global properties of an improved hepatitis B virus model," Nonlinear Analysis: Real World Applications, vol. 11, no. 4, pp. 3131-3138, 2010.

[12] M. A. Safi and A. B. Gumel, "The effect of incidence functions on the dynamics of a quarantine/isolation model with time delay," Nonlinear Analysis: Real World Applications, vol. 12, no. 1, pp. 215-235, 2011.

[13] A. Rachah and D. F. M. Torres, "Mathematical modelling, simulation, and optimal control of the 2014 Ebola outbreak in West Africa," Discrete Dynamics in Nature and Society, vol. 2015, Article ID 842792, 9 pages, 2015.

[14] H. S. Rodrigues, M. T. T. Monteiro, and D. F. M. Torres, "Vaccination models and optimal control strategies to dengue," Mathematical Biosciences, vol. 247, pp. 1-12, 2014.

[15] P. Rodrigues, C. J. Silva, and D. F. M. Torres, "Cost-effectiveness analysis of optimal control measures for tuberculosis," Bulletin of Mathematical Biology, vol. 76, no. 10, pp. 2627-2645, 2014.

[16] R. Anderson and R. May, Infectious Diseases Of Humans: Dynamics And Control, Oxford University Press, Oxford, UK, 1992.

[17] J. R. Williams, D. J. Nokes, G. F. Medley, and R. M. Anderson, "The transmission dynamics of hepatitis B in the UK: a mathematical model for evaluating costs and effectiveness of immunization programmes," Epidemiology and Infection, vol. 116, no. 1, pp. 71-89, 1996.

[18] G. F. Medley, N. A. Lindop, W. J. Edmunds, and D. J. Nokes, "Hepatitis-B virus endemicity: heterogeneity, catastrophic 
dynamics and control," Nature Medicine, vol. 7, no. 5, pp. 619-624, 2001.

[19] S. Zhao, Z. Xu, and Y. Lu, "A mathematical model of hepatitis $B$ virus transmission and its application for vaccination strategy in China," International Journal of Epidemiology, vol. 29, no. 4, pp. 744-752, 2000.

[20] E. A. Bakare, A. Nwagwo, and E. Danso-Addo, "Optimal control analysis of an sir epidemic model with constant recruitment," International Journal of Applied Mathematical Research, vol. 3, no. 3, p. 273, 2014.

[21] A. V. Kamyad, R. Akbari, A. A. Heydari, and A. Heydari, "Mathematical modeling of transmission dynamics and optimal control of vaccination and treatment for hepatitis b virus," Computational and Mathematical Methods in Medicine, vol. 2014, Article ID 475451, 15 pages, 2014.

[22] N. O. Onyango, "Multiple endemic solutions in an epidemic hepatitis B model without vertical transmission," Applied Mathematics, vol. 2014, Article ID 516242, 2014.

[23] T. Zhang, K. Wang, and X. Zhang, "Modeling and analyzing the transmission dynamics of hbv epidemic in xinjiang, China," PloS one, vol. 10, no. 9, Article ID e0138765, 2015.

[24] T. Khan and G. Zaman, "Classification of different hepatitis B infected individuals with saturated incidence rate," SpringerPlus, vol. 5, no. 1, p. 1082, 2016.

[25] T. Khan, G. Zaman, and M. I. Chohan, "The transmission dynamic and optimal control of acute and chronic hepatitis B," Journal of Biological Dynamics, vol. 11, no. 1, pp. 172-189, 2017.

[26] S. Nana-Kyere, J. Ackora-Prah, E. Okyere, S. Marmah, and T. Afram, "Hepatitis b optimal control model with vertical transmission," Applications of Mathematics, vol. 7, no. 1, pp. 5-13, 2017.

[27] A. Atangana, "Modelling the spread of covid-19 with new fractal-fractional operators: can the lockdown save mankind before vaccination?" Chaos, Solitons \& Fractals, vol. 136, Article ID 109860, 2020.

[28] A. Atangana and S. İğret Araz, "Nonlinear equations with global differential and integral operators: existence, uniqueness with application to epidemiology," Results in Physics, vol. 20, Article ID 103593, 2021.

[29] S. G. Samko, Fractional Integrals and Derivatives, Theory and Applications, Minsk; Nauka I Tekhnika, New York, NY, USA, 1987.

[30] D. Baleanu, Z. B. Güvenç, and J. T. Machado, New Trends in Nanotechnology and Fractional Calculus Applications, Springer, Manhattan, NY, USA, 2010.

[31] X. Yang, L. Chen, and J. Chen, "Permanence and positive periodic solution for the single-species nonautonomous delay diffusive models," Computers \& Mathematics with Applications, vol. 32, no. 4, pp. 109-116, 1996.

[32] L. Kexue and P. Jigen, "Laplace transform and fractional differential equations," Applied Mathematics Letters, vol. 24, no. 12, pp. 2019-2023, 2011.

[33] W. Lin, "Global existence theory and chaos control of fractional differential equations," Journal of Mathematical Analysis and Applications, vol. 332, no. 1, pp. 709-726, 2007.

[34] F. Wang and Y. Yang, "Correction: fractional order BARBALAT'S lemma and its applications in the stability of fractional order nonlinear systems," Mathematical Modelling and Analysis, vol. 22, no. 4, pp. 503-513, 2017.

[35] K. Hattaf, N. Yousfi, and A. Tridane, "Mathematical analysis of a virus dynamics model with general incidence rate and cure rate," Nonlinear Analysis: Real World Applications, vol. 13, no. 4, pp. 1866-1872, 2012.
[36] H. Ramos, Z. Kalogiratou, T. Monovasilis, and T. E. Simos, "An optimized two-step hybrid block method for solving general second order initial-value problems," Numerical Algorithms, vol. 72, no. 4, pp. 1089-1102, 2016.

[37] D. Baleanu, A. Jajarmi, and M. Hajipour, "On the nonlinear dynamical systems within the generalized fractional derivatives with Mittag-Leffler kernel," Nonlinear Dynamics, vol. 94, no. 1, pp. 397-414, 2018.

[38] C. Li and F. Zeng, Numerical Methods for Fractional Calculus, Chapman and Hall/CRC, Boca Raton, FL, USA, 2019. 